\title{
Cardioprotective GLP-1 metabolite prevents ischemic cardiac injury by inhibiting mitochondrial trifunctional protein- $\alpha$
}

M. Ahsan Siraj, ${ }^{1,2}$ Dhanwantee Mundil, ${ }^{2,3}$ Sanja Beca, ${ }^{4}$ Abdul Momen, ${ }^{2}$ Eric A. Shikatani, ${ }^{2,5}$ Talat Afroze, ${ }^{2}$ Xuetao Sun, ${ }^{2}$ Ying Liu, ${ }^{2}$ Siavash Ghaffari, ${ }^{6}$ Warren Lee, ${ }^{5,6,7,8}$ Michael B. Wheeler, ${ }^{2,3}$ Gordon Keller, ${ }^{9,10}$ Peter Backx, ${ }^{2}$ and Mansoor Husain ${ }^{1,2,3,4,5,8,9,10,11}$

${ }^{1}$ Ted Rogers Centre for Heart Research, University of Toronto, Toronto, Ontario, Canada. ${ }^{2}$ Toronto General Hospital Research Institute, University Health Network, Toronto, Ontario, Canada. ${ }^{3}$ Department of Physiology, University of Toronto, Toronto, Ontario, Canada. ${ }^{4}$ Heart and Stroke Richard Lewar Center of Excellence in Cardiovascular Research, and ${ }^{5}$ Laboratory Medicine and Pathobiology, University of Toronto, Toronto, Ontario, Canada. ${ }^{6}$ Keenan Research Centre for Biomedical Research, St. Michael's Hospital, Toronto, Ontario, Canada. ${ }^{7}$ Department of Biochemistry, ${ }^{8}$ Department of Medicine, and ${ }^{9}$ Department of Medical Biophysics, University of Toronto, Toronto, Ontario, Canada. ${ }^{10}$ McEwen Centre for Regenerative Medicine, and ${ }^{11}$ Peter Munk Cardiac Centre, University Health Network, Toronto, Ontario, Canada.

\begin{abstract}
Mechanisms mediating the cardioprotective actions of glucagon-like peptide 1 (GLP-1) were unknown. Here, we show in both ex vivo and in vivo models of ischemic injury that treatment with CLP-1(28-36), a neutral endopeptidase-generated (NEPgenerated) metabolite of CLP-1, was as cardioprotective as CLP-1 and was abolished by scrambling its amino acid sequence. CLP-1(28-36) enters human coronary artery endothelial cells (caECs) through macropinocytosis and acts directly on mouse and human coronary artery smooth muscle cells (caSMCs) and caECs, resulting in soluble adenylyl cyclase Adcy10-dependent (sAC-dependent) increases in CAMP, activation of protein kinase A, and cytoprotection from oxidative injury. GLP-1(28-36) modulates SAC by increasing intracellular ATP levels, with accompanying CAMP accumulation lost in $s A C^{-/-}$cells. We identify mitochondrial trifunctional protein- $\alpha$ (MTP $\alpha$ ) as a binding partner of CLP-1(28-36) and demonstrate that the ability of CLP-1(2836) to shift substrate utilization from oxygen-consuming fatty acid metabolism toward oxygen-sparing glycolysis and glucose oxidation and to increase cAMP levels is dependent on MTP $\alpha$. NEP inhibition with sacubitril blunted the ability of GLP-1 to increase CAMP levels in coronary vascular cells in vitro. GLP-1(28-36) is a small peptide that targets novel molecular (MTPa and SAC) and cellular (caSMC and caEC) mechanisms in myocardial ischemic injury.
\end{abstract}

\section{Introduction}

In myocardial infarction (MI), infarct size is the most determinant cause of morbidity and mortality (1). Although early reperfusion is of unequivocal benefit, this treatment is inherently complicated by ischemia reperfusion injury (IRI) (2). Indeed, as much as $50 \%$ of post-MI myocardial injury is attributable to IRI (3). As such, therapies aimed at limiting IRI have potential for clinical impact. Although many treatments effective in animal models of IRI have failed to translate into clinical use, glucagon-like peptide 1 (GLP-1) and the GLP-1 receptor (GLP-1R) agonist exenatide have shown benefits in IRI in both animal models (4-7) and small early-phase clinical trials $(8,9)$.

The predominant active form of GLP-1 is 30-amino acid GLP-1 (7-36) amide (also known as GLP-1), which acts through a stimulatory G protein-coupled (Gs-coupled) GLP-1R. However GLP-1 is rapidly degraded into the noninsulinotropic 28 -amino acid metab-

\section{Authorship note: MAS and DM contributed equally to this work}

Conflict of interest: $\mathrm{MH}$ has provided consultancy services to AstraZeneca, Novo Nordisk, Merck \& Co., and Hoffman-La Roche with respect to incretin-targeted therapeutics and has received investigator-initiated research grants from Novo Nordisk and Merck \& Co. Portions of this work underlie a provisional patent application (PCT/ CA2013/000920; "Peptides and methods for preventing ischemic tissue injury"). Copyright: (c) 2020, American Society for Clinical Investigation.

Submitted: February 27, 2018; Accepted: November 13, 2019; Published: January 27, 2020. Reference information: J Clin Invest. 2020;130(3):1392-1404.

https://doi.org/10.1172/JCI99934 olite GLP-1(9-36) by ubiquitous expression of dipeptidyl peptidase 4 (DPP-4) (10). GLP-1 and GLP-1(9-36) are also cleaved by neutral endopeptidase (NEP24.11), yielding GLP-1(28-36), a 9-amino acid carboxy-terminal peptide with biological activity (11).

At present, several DPP-4-resistant GLP-1R agonists and numerous DPP-4 inhibitors (DPP-4i) have been approved for the treatment of type 2 diabetes (T2D) and are widely used. The GLP-1R agonist liraglutide has also received regulatory approval for the treatment of obesity and conferred protection in an obesity-associated animal model of cardiomyopathy (12). Moreover, in subjects with diabetes who were at high risk of cardiovascular events, treatment with GLP-1R agonists based on the structure of endogenous GLP-1, namely liraglutide, semaglutide, albiglutide, and dulaglutide, has demonstrated reductions in major adverse cardiovascular events (MACEs) that appear to be unrelated to the glycemic effects of these agents (13-16). By contrast, treatment of high-risk diabetic patients with GLP-1R agonists such as lixisenatide and exenatide, which are based on the structure of nonhuman exendin 4, has not resulted in consistent reductions in MACEs $(17,18)$. Whether these differences are attributable in part to molecular structures that exclude the formation of bioactive metabolites has not been addressed.

With regard to the utility of GLP-1R agonists in myocardial IRI, the first report by Nikolaidis et al. was a nonrandomized, unblinded study involving 10 treated (vs. 11 untreated) patients, in whom a 72-hour i.v. infusion of GLP-1 following reperfusion 
was shown to improve cardiac function (19). Similar effects of GLP-1 were reported in another group of 20 nondiabetic patients with normal cardiac function undergoing elective angioplasty (9). Subsequently, a randomized, placebo-controlled, double-blinded study involving 74 patients undergoing primary angioplasty for acute MI showed that a 6-hour i.v. infusion of exenatide reduced infarct size as a function of area at risk (AAR) (8).

However, the mechanisms underlying these effects have not been fully elucidated, with some preclinical studies revealing inconsistent results (20). For example, we observed that both GLP-1 and its presumed "inert" metabolite GLP-1(9-36) showed coronary and mesenteric artery vasodilation, as well as cardioprotection from IRI ex vivo, in isolated tissues from both WT and Glp1r-KO (Glp1r/-) mice, whereas the nondegradable GLP-1R agonist exenatide did not have these effects in the absence of a functional GLP-1R $(4,21)$. This suggested either the existence of an alternate receptor for GLP-1 and/or GLP-1(9-36), or receptorindependent mechanisms of action. Subsequently, mice with cardiomyocyte-specific (CM-specific) deletion of Glp1r also showed cardioprotective effects of liraglutide in an in vivo model of MI (22), suggesting that the cardioprotective effects of this agent are derived from actions independent of CMs and/or the GLP-1R.

Here, we show direct cardiovascular effects of the GLP$1(28-36)$ peptide. In both in vivo and ex vivo models of myocardial ischemic injury, GLP-1(28-36) prevented cardiac dysfunction, reduced infarct size, and protected coronary vascular cells from oxidative stress injury. We show that the cardioprotective actions of GLP-1(28-36) do not depend on a functional transmembrane GLP-1R but rather are mediated intracellularly through type 10 soluble adenylyl cyclase (sAC), accompanied by increased cAMP levels, protein kinase A (PKA) activation, and endothelial nitric oxide synthase (eNOS) phosphorylation. We further demonstrate that GLP-1(28-36) activates SAC and increases cAMP levels by increasing intracellular levels of ATP in mouse and human coronary artery smooth muscle cells (caSMCs) and human coronary artery endothelial cells (caECs), but not mouse or human CMs. Finally, to begin to understand how GLP-1(28-36) achieves its effects on intracellular ATP, we conducted an unbiased proteomic analysis of heart proteins capable of binding a biotinylated (but still functional) GLP-1(28-36). This revealed an interaction between the metabolite and mitochondrial trifunctional protein- $\alpha(\mathrm{MTP} \alpha)$, which is known to regulate fatty acid oxidation (FAO) (23). Cell metabolism experiments revealed an MTP $\alpha$-dependent ability of GLP-1(28-36) to shift substrate utilization away from FAO to more efficient glucose oxidation and higher levels of cAMP. Together, our studies demonstrate that GLP-1(28-36) protects the heart from IRI by activating sAC through an oxygen-sparing substrate shift mediated by MTP $\alpha$, with reductions in metabolic oxidative stress.

\section{Results}

Pretreatment with GLP-1(28-36) reduces myocardial infarct size in mice. We first tested the therapeutic relevance of GLP-1(28-36) in vivo in 10- to 12 -week-old male $\mathrm{C} 57 \mathrm{BL} / 6 \mathrm{~J}$ mice subjected to permanent ligation of the left anterior descending (LAD) artery following 14 days of s.c. infusions of GLP-1(28-36) (18.5 nmol/ $\mathrm{kg} / \mathrm{d})(24)$, a scrambled amino acid sequence of GLP-1(28-36) [scrambled(28-36), $18.5 \mathrm{nmol} / \mathrm{kg} / \mathrm{d}$; negative control], saline, or GLP-1 (3.5 pmol $/ \mathrm{kg} / \mathrm{min}$; positive control) (Figure $1 \mathrm{~A}$ ). Heart sections stained with 2,3,5-triphenyltetrazolium chloride (TTC) harvested 4 days after MI revealed obvious reductions in unstained infarct areas of GLP-1(28-36)-treated hearts as compared with the scramble- or saline-treated controls (Figure 1B). Blinded histomorphometry confirmed that pretreatment with GLP-1(28-36) significantly decreased infarct size 4 days after MI as compared with scramble- and saline-treated controls $(24.9 \%$ $\pm 2.4 \%, n=7$, vs. scramble: $32.5 \% \pm 1.8 \%, n=7$; saline: $34.3 \% \pm$ $2.8 \% n=9 ; P<0.05$ for both) (Figure 1C), with the effect of GLP1(28-36) treatment being comparable to that of GLP-1 $(23.0 \% \pm$ $1.9 \%, n=13 ; P=\mathrm{NS})$.

Given that infusions of GLP-1(28-36) and GLP-1 have been shown to influence body weight and blood glucose levels in highfat diet-fed mice (24) and that we ourselves have documented an effect of calorie restriction and weight loss on cardioprotection (25), we examined the consequence of our 14-day infusions on these parameters. Under ad libitum feeding conditions, we found that heart, body, and heart/body weights and nonfasting tail blood glucose measurements were not affected by treatment assignment (Supplemental Figure 1; supplemental material available online with this article; https://doi.org/10.1172/JCI99934DS1), suggesting that the observed reductions in infarct size were unrelated to changes in body weight or glycemic control.

The direct cardioprotective effects of GLP-1(28-36) do not depend on the GLP-1R. To assess whether GLP-1(28-36) can exert direct cardiac effects, we used an ex vivo isolated heart global IRI model under constant coronary perfusion pressure (4) (Figure 1D). Representative left ventricular developed pressure (LVDP) tracings showed that a 20-minute perfusion with GLP-1(28-36) (6 nM) improved recovery of LVDP following IRI as compared with bufferand scramble-treated $(6 \mathrm{nM})$ controls (Figure 1E). Of note, the dose of GLP-1(28-36) used was selected on the basis of its dose response effect on post-IRI LVDP (Supplemental Figure 2). Recovery of LVDP was greater in GLP-1(28-36) hearts than in buffer- or scrambletreated hearts $(57.6 \% \pm 6.6 \%, n=12$ vs. buffer: $22.7 \% \pm 3.6 \%, n=13$; scramble: $35.9 \% \pm 5.6 \%, n=4 ; P<0.05$ for both comparisons) and comparable to GLP-1 $(67.3 \% \pm 8.6 \%, n=13 ; P=\mathrm{NS})$ (Figure $1 \mathrm{~F}$, gray bars). Pretreatment with GLP-1(28-36) also reduced lactate dehydrogenase $(\mathrm{LDH})$ release into coronary effluent during reperfusion as compared with buffer- or scramble-treated controls (Figure $1 G$ ).

To address the question of whether the cardioprotective actions of GLP-1(28-36) require a functional transmembrane GLP-1R, we repeated these experiments using hearts isolated from mice with genetic deletion of 2 exons of the Glp1r gene (26). These studies revealed that the cardioprotective actions of GLP-1(28-36) were preserved in hearts from Glp1r/- mice. As observed previously (4), the parent peptide GLP-1 also retained its ability to protect the heart from IRI in the absence of a functional GLP-1R (Figure 1F, white bars).

The cardioprotective actions of GLP-1(28-36) require sAC. To determine the GLP-1R-independent actions of GLP-1(28-36), we next examined the involvement of second messenger cAMP. Studies in pancreatic $\beta$ cells had demonstrated that GLP-1(28-36) stimulates cAMP/PKA (27), a key upstream signaling pathway in pharmacological and ischemic preconditioning (28). Given the increasing evidence of alternative receptor-independent sources of cAMP (from intracellular SAC) $(29,30)$, we hypothesized that 


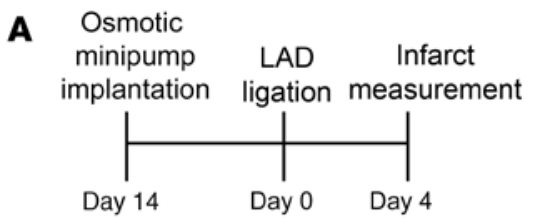

B
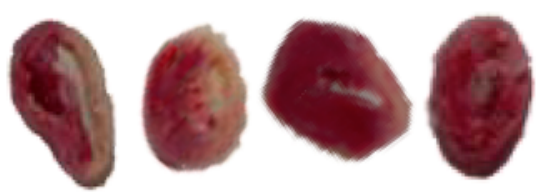

Saline

Scram

28-36

GLP-1

\begin{tabular}{|c|c|c|c|} 
Equilibrium & Perfusion & Ischemia & Reperfusion \\
\hline $20 \mathrm{~min}$ & $40 \mathrm{~min}$ & $30 \mathrm{~min}$ & $40 \mathrm{~min}$
\end{tabular}

E

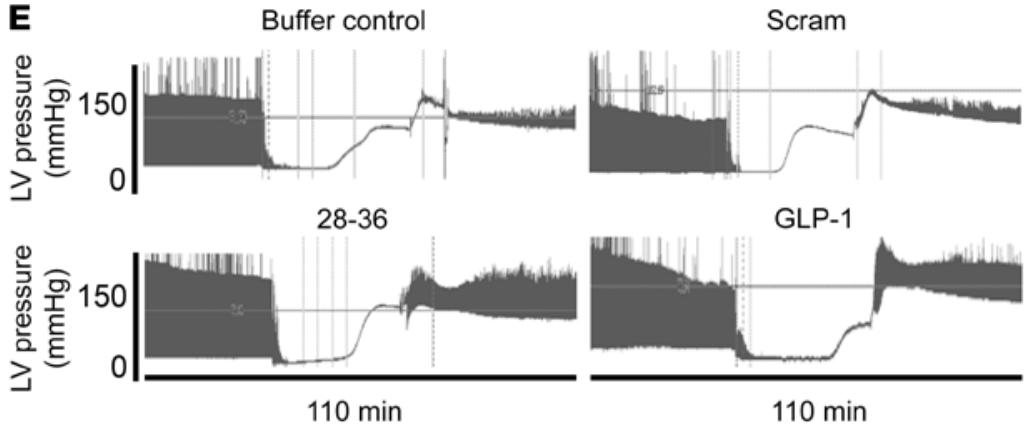

F

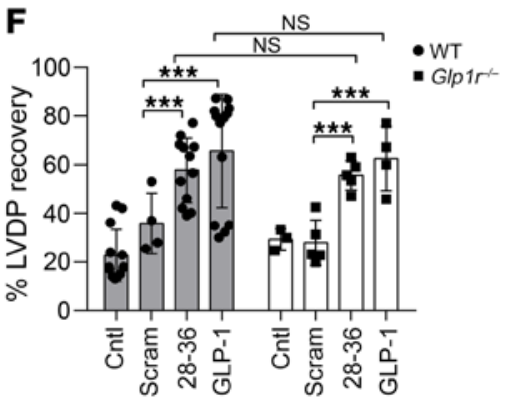

G

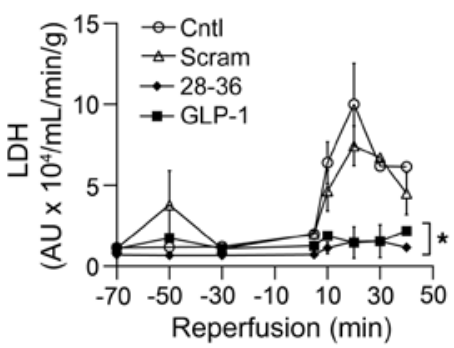

Figure 1. Pretreatment with GLP-1(28-36) reduces infarct size in mice and provides direct GLP-1R-independent cardioprotection in isolated mouse hearts. (A) Schematic of the in vivo animal protocol. (B) Representative photomicrographs of TTC-stained heart sections show infarcted (white) versus viable (red) tissue on day 4 after MI. Smaller infarct areas were observed in hearts treated with GLP-1(28-36) or GLP-1 (positive controls) as compared with hearts treated with saline or scrambled(28-36) (Scram) (negative controls). (C) Grouped data showing quantification of infarct size as a percentage of LV surface area on day 4 after MI in WT mice pretreated for 14 days with saline $(n=9)$, scrambled(28-36) (both $18.5 \mathrm{nmol} / \mathrm{kg} / \mathrm{day} ; n=7$ ), GLP-1(28-36), or GLP-1 (3.5 pmol/kg/min; $n=13$ ). (D) IRI protocol of retrograde, nonrecirculating Langendorff perfusion of isolated hearts from male 10- to 12-week-old WT or GIp1r-1- mice. (E) Representative tracings showing LVDP recordings from isolated, perfused WT hearts treated with GLP-1(28-36) or GLP-1 or with buffer only or scrambled(28-36) (Scram) controls. (F) LVDP recovery expressed as a percentage of LVDP at the end of reperfusion over LVDP before ischemia. LVDP recovery is shown in hearts perfused with $6 \mathrm{nM} \mathrm{GLP-1(28-36),} \mathrm{scrambled(28-36)} \mathrm{control,} \mathrm{or} \mathrm{buffer-only} \mathrm{control,} \mathrm{or} \mathrm{with} \mathrm{0.3} \mathrm{nM} \mathrm{GLP-1} \mathrm{(} n=4-13$ WT mice/group; gray bars; $n=3-5 \mathrm{Clp} 7 r^{-1-}$ mice/group; white bars). (C) LDH release into coronary effluents from perfused WT mouse hearts, measured by ELISA at timed intervals between 10 and 40 minutes of reperfusion and normalized to coronary flow ( $\mathrm{mL} / \mathrm{min} / \mathrm{g}$ heart weight) $(n=3)$. Data represent the mean \pm SEM. ${ }^{*} P<0.05$ and ${ }^{* * *} P<0.001$ versus the corresponding control, by 1-way ANOVA with Bonferroni's post hoc test. Cntl, control.

GLP-1(28-36) mediates its GLP-1R-independent effects through sAC. We first probed for sAC expression by immunoblotting and detected abundant protein expression in mouse hearts and specific heart chambers (Figure 2A). A more in-depth examination revealed abundant sAC expression in caSMCs and caECs compared with expression in CMs (Supplemental Figure 3A). Our inability to detect sAC in tissues from sAC-KO mice $\left(s A C^{-/-}\right)$attested to the specificity of the sAC antibody (Supplemental Figure 3B).

Coronary effluents of isolated mouse hearts perfused with GLP1(28-36) showed a modest increase in extracellular cAMP levels that did not differ from levels in controls (Figure 2B). However, in isolated mouse hearts perfused with the sAC inhibitor $\mathrm{KH}$, we observed loss of cardioprotection with GLP-1(28-36), but not GLP-1, as measured by LVDP at the end of the reperfusion $(25.2 \pm 1.7 \mathrm{mmHg}, n=3$, vs. $73.1 \pm 5.3 \mathrm{mmHg}, n=3: P<0.0001)$. On the other hand, perfusion of isolated hearts with $2^{\prime} 5^{\prime}$-dideoxyadenosine (Ddox) to inhibit transmembrane AC (tmAC) did not affect the cardioprotective actions of GLP-1(28-36), but did reduce the cardioprotective effect of GLP-
1 (67.6 $\pm 5.8 \mathrm{mmHg}, n=3$, vs. $27.1 \pm 5.3 \mathrm{mmHg}, n=3 ; P<0.0001)$. The scrambled peptide control did not produce any recovery of $\mathrm{LV}$ function, which was not affected by the inhibitors of SAC (36.4 \pm $5.2 \mathrm{mmHg}, n=3 ; P=\mathrm{NS})$ or tmAC $(37.6 \pm 5.3 \mathrm{mmHg}, n=3 ; P=\mathrm{NS})$ (Figure 2, C and D). These data suggest that although the cardioprotective actions of GLP-1 in the isolated mouse heart require tmAC, those of GLP-1(28-36) depend solely on sAC. Given the previously observed cardioprotective actions of GLP-1 in the absence of the GLP-1R, these data suggest that the ex vivo heart may not effectively metabolize GLP-1 to GLP-1(28-36). More important, the concentration of GLP-1(28-36) required for cardioprotection (6 nM) could not possibly be generated by degradation of the 20 -fold lower amounts of the parent GLP-1 used in our experiments $(0.3 \mathrm{nM})$.

The cytoprotective effects of GLP-1(28-36) are attenuated in the absence of SAC. To test the importance of SAC to the actions of GLP$1(28-36)$, we used an in vivo IRI model in $s A C^{-/-}$mice and compared this with WT littermate controls. We treated 10- to 12-week-old male $s A C^{-/-}$mice with intrajugular $3.6 \mu \mathrm{mol} / \mathrm{kg}$ GLP-1(28-36) versus 
A

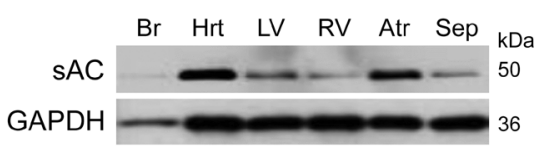

B

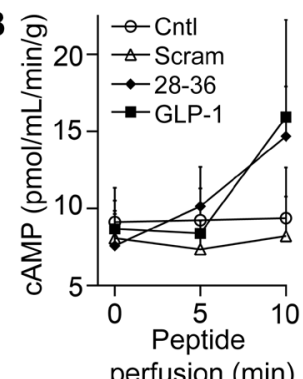

C

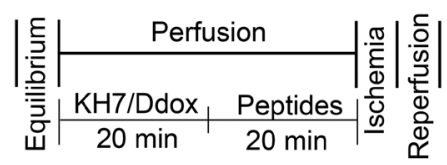

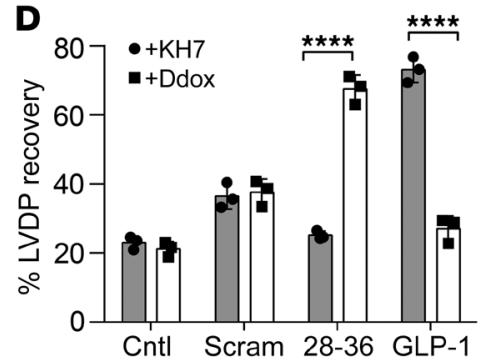

E

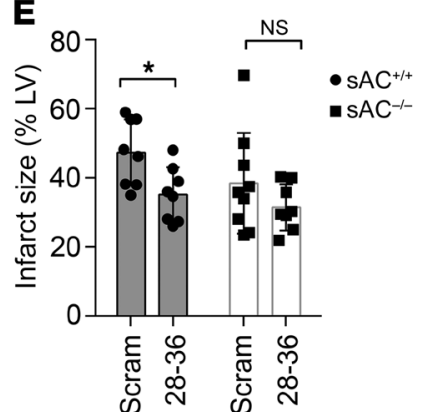

H

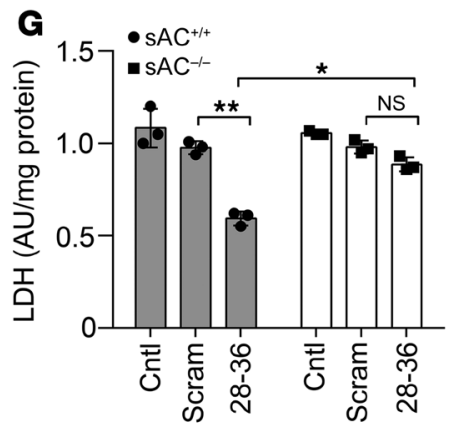

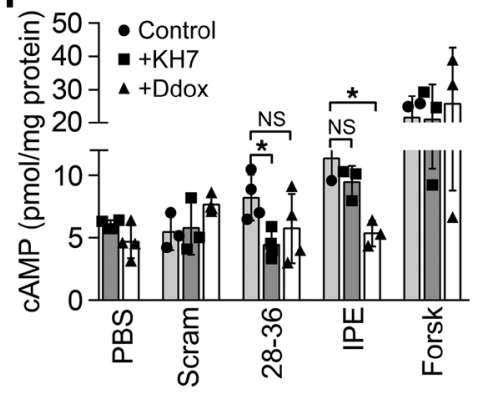

$\mathbf{F}$

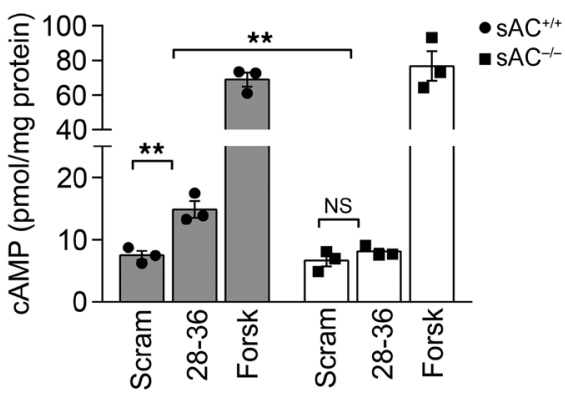

I

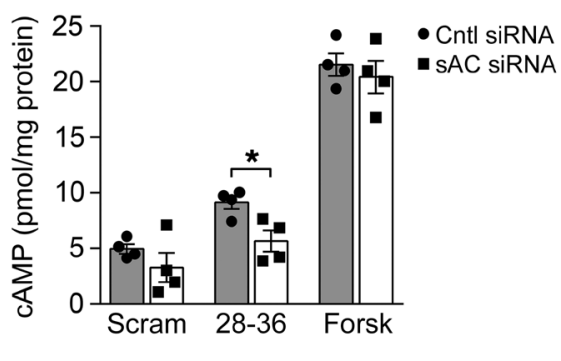

Figure 2. The cardioprotective effects of GLP-1(28-36) are sAC dependent. (A) Representative Western blot of 50-kDa sAC isoform expression in WT mouse lysates of whole heart (Hrt), LV, right ventricle (RV), atria (Atr), septum (Sep), and brain (Br, negative control). GAPDH was used as a loading control $(n=3)$.(B) cAMP levels in coronary effluents were measured at timed intervals during an initial 10-minute perfusion of WT hearts with either $6 \mathrm{nM}$ GLP-

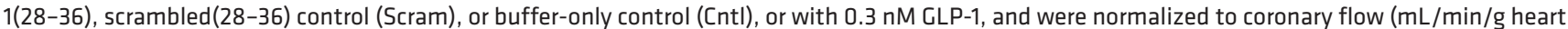
weight). (C) Schematic of IRI protocol and (D) percentage of LVDP recovery of isolated WT mouse hearts with the sAC inhibitor KH7 (20 $\mu \mathrm{mol} / \mathrm{L}$ ) or the tmAC inhibitor Ddox (50 $\mu \mathrm{mol} / \mathrm{L})$ prior to perfusion with buffer only (Cntl), scrambled(28-36), GLP-1(28-36), or GLP-1 ( $n=3 /$ group). (E) Quantification of infarct size as a percentage of the AAR on day 2 after in vivo IRI in WT $\left(s A C^{+/+}\right)$mice $\left(n=8 /\right.$ group, gray bars) and $s A C^{-/-}$littermates $(n=8-9 /$ group, , white bars). (F) Intracellular cAMP accumulation after treatment with GLP-1(28-36) in caSMCs isolated from $s A C^{-/-}$mice (white bars) as compared with $s A C^{+/+}$

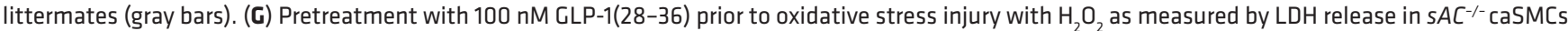
(white bars) versus $S A C^{+/+}$control caSMCs (gray bars). (H) Intracellular cAMP accumulation in human caSMCs treated with the $s A C$ inhibitor KH7 (25 $\mu M$, dark gray bars), the tmAC inhibitor Ddox ( $50 \mu \mathrm{M}$, white bars), or PBS (light gray bars) for 3 hours followed by a 15-minute treatment with GLP-1(28-36), scrambled control, IPE, or forskolin. Intracellular CAMP accumulation was normalized to the total cellular protein concentration. (I) CAMP levels in cell lysates were determined following pretreatment with $100 \mathrm{nM}$ each of GLP-1(28-36), scrambled control, or forskolin in siRNA-treated cells (white bars) versus the scrambled siRNA control (gray bars) ( $n=3$ /group for all cAMP and LDH assays, each in triplicate). Data represent the mean \pm SEM. ${ }^{*} P<0.05$, ${ }^{* *} P<0.01$, and ${ }^{* * *} P<0.0001$ versus the corresponding control by 2-way ANOVA with Bonferroni's post hoc test. Forsk, forskolin.

the scrambled control 30 minutes prior to standard open-chest IRI involving a 45-minute LAD occlusion. The hearts were removed on day 2 after IRI for TTC staining to measure infarct size. In addition, Evan's blue dye was injected to establish the AAR after re-ligating the LAD at the original site. Blinded histomorphometric analysis revealed that GLP-1(28-36) did not decrease the infarct size in $s A^{-/-}$ mice compared with the scramble-treated control mice [GLP-1(28$36): 31.5 \% \pm 3.1 \%, n=8$, vs. scramble: $38.3 \% \pm 5.9 \%, n=9 ; P=\mathrm{NS}]$, whereas the cardioprotective effects were preserved in WT hearts [GLP-1(28-36): $35.1 \% \pm 3.1 \%, n=5$, vs. scramble: $47.4 \% \pm 3.9 \%, n=$ $5 ; P<0.05]$ (Figure 2E).
Given that GLP-1 biology is complex and may involve several parallel mechanisms with or without transmembrane receptors (31) and distinct metabolites (32), we chose to next focus on the direct actions of the single metabolite GLP-1(28-36) using in-depth in vitro studies into its cellular and molecular actions. We began by investigating sAC-dependent molecular actions of GLP-1(28-36) in caSMCs. The ability of GLP-1(28-36) to stimulate cAMP generation was lost in caSMCs isolated from $s A C^{-/-}$ mice [GLP-1(28-36): $8.1 \pm 0.5$ vs. scramble: $6.6 \pm 0.6, n=3 ; P=$ NS] but not from WT mice [GLP-1(28-36): $14.9 \pm 1.3$ vs. scramble: $7.5 \pm 0.8, n=3 ; P<0.01]$ ( $\mathrm{pmol} / \mathrm{mg}$ protein; Figure $2 \mathrm{~F}$ ). Moreover, 
A

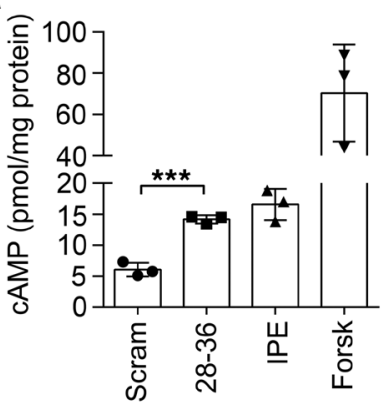

D

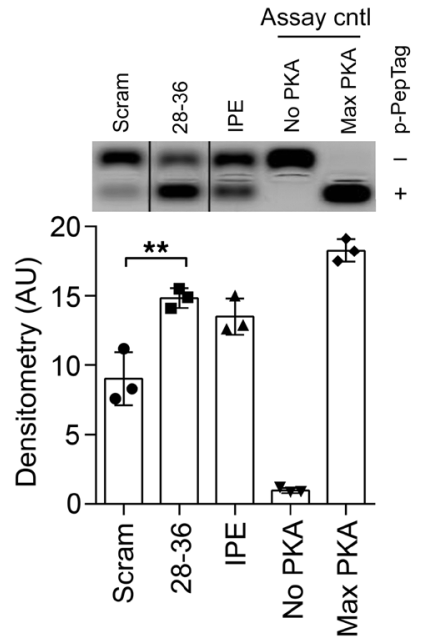

B

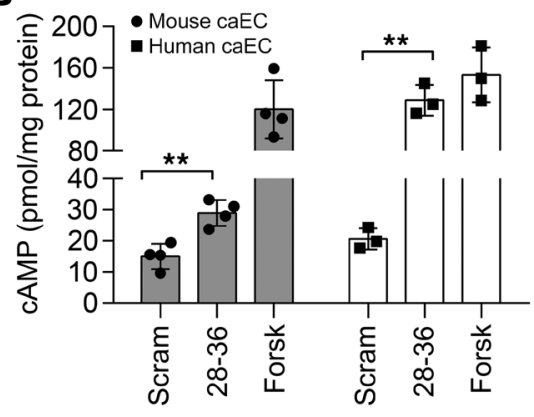

E
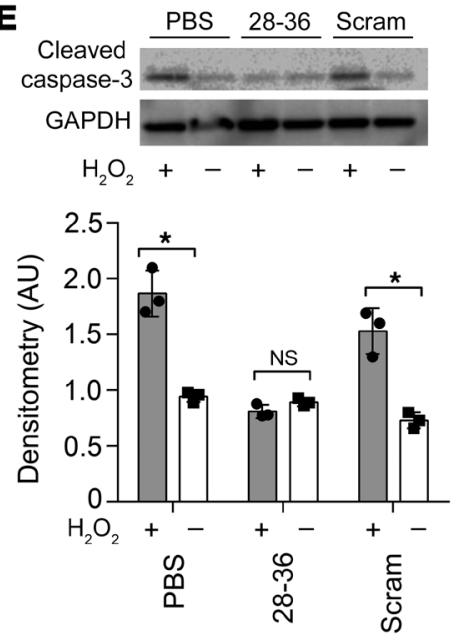

C

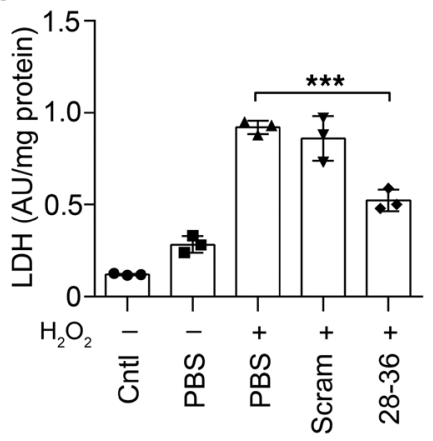

Figure 3. GLP-1(28-36) stimulates intracellular cAMP accumulation, activates PKA, and prevents cytotoxicity in vascular cells. (A and B) Intracellular cAMP accumulation measured by enzymatic immunoassay in lysates from (A) caSMCs and (B) mouse and human caECs treated for 10 minutes with 100 nM each of GLP-1(28-36), scrambled control, IPE, forskolin, or PBS control ( $n=3 /$ treatment, each in triplicate). (C) Mouse caSMCs were pretreated with $100 \mathrm{nM} \mathrm{GLP-1}(28-36)$ or scrambled control for 20 minutes followed by incubation with $\mathrm{H}_{2} \mathrm{O}_{2}(100 \mu \mathrm{M})$ for 48 hours to induce oxidative stress ( $n=3 /$ each, in triplicate). LDH release was assayed by ELISA in duplicates from aliquots of cell culture media. (D) Mouse caSMCs were treated as above and lysed with PKA lysis buffer, with PKA activity determined by separation of phosphorylated (+ p-Peptag) and nonphosphorylated (- p-Peptag) substrate using electrophoresis. Densitometric analysis of phosphorylated bands showed increased PKA activity with GLP-1(28-36) as compared with the scrambled control. The blot is representative of 3 independent experiments. Max, maximum. (E) Whole-cell lysates were extracted from mouse caSMCs undergoing a similar 20-minute peptide pretreatment schedule, and apoptosis was assessed by cleaved caspase-3 activation at the end of the 7-hour incubation with or without $\mathrm{H}_{2} \mathrm{O}_{2}(100 \mu \mathrm{M})$. Representative Western blot and normalized densitometric analysis ( $n=3 /$ group) show that GLP-1(28-36) prevented activation of cleaved caspase-3 in the presence of $\mathrm{H}_{2} \mathrm{O}_{2}$ versus controls. Data represent the mean $\pm \mathrm{SEM}$. ${ }^{*} P<0.05$, ${ }^{*} P<0.01$, and ${ }^{* * *} P<0.001$, by 1 -way ANOVA (except for data in $\mathbf{E}$, which were analyzed by 2-way ANOVA); NS versus the corresponding control with Bonferroni's post hoc test.

we found that GLP-1(28-36) failed to protect $s A C^{-/}$caSMCs from oxidative stress injury, as evidenced by nonsignificant decreases in LDH release versus the scrambled control $(0.74 \pm 0.03$ vs. 0.83 $\pm 0.03, n=3 ; P=\mathrm{NS})$ and compared with WT ( $0.51 \pm 0.02$ vs. 0.78 $\pm 0.04, n=3 ; P<0.01$ ) (Figure 2G). Regrettably, we were not able to reliably culture mouse caECs or mouse cardiac microvascular ECs from WT or $s A C^{-/-}$mice.

GLP-1(28-36) enters coronary vascular cells, increases cAMP-PKA activity, and prevents cytotoxicity. We surveyed specific cardiovascular cell types and found that the effect of GLP-1(28-36) on intracellular cAMP levels in both atrial and ventricular CMs was no different than that of the scrambled control (Supplemental Figure 4A). This inability of GLP-1(28-36) to increase cAMP levels in CMs was preserved in human embryonic stem cell-derived CMs (hESC-CMs) (Supplemental Figure 4B). By contrast, even a brief (10-minute) incubation with GLP-1(28-36) caused a 2.3- \pm 0 .4-fold increase in intracellular cAMP levels in mouse caSMCs as compared with treat- ment with the scrambled control $(P<0.0001)$. In this cell type, the effect of GLP-1(28-36) on cAMP levels was as robust as that of the adrenergic agonist isoproterenol (IPE) (Figure 3A). In commercially available mouse and human primary caECs, we also observed robust cAMP responses to GLP-1(28-36) (Figure 3B).

To visualize whether GLP-1(28-36) undergoes cellular uptake, we synthesized a biotinylated GLP-1(28-36), placing biotin at the C-terminus of the peptide via a lysine link. Consistent with rapid generation of cAMP in response to this peptide, biotinylated GLP-1(2836) entered caSMCs with the strongest intracellular signals observed within 15 minutes (Supplemental Figure 5). To elucidate the mechanism of cellular uptake of GLP-1(28-36), we also conjugated fluorescein fluorophore (FAM) with GLP-1(28-36) and incubated human caECs with FAM(28-36) (250 nM) for 15 minutes. We found that the endocytosis and dynamin inhibitor dyngo- $4 \mathrm{~A}(30 \mu \mathrm{M})(33)$ abrogated the internalization of $\operatorname{FAM}(28-36)$, implicating endocytosis as a method of cellular uptake of GLP-1(28-36) (Supplemental Figure 6, A 

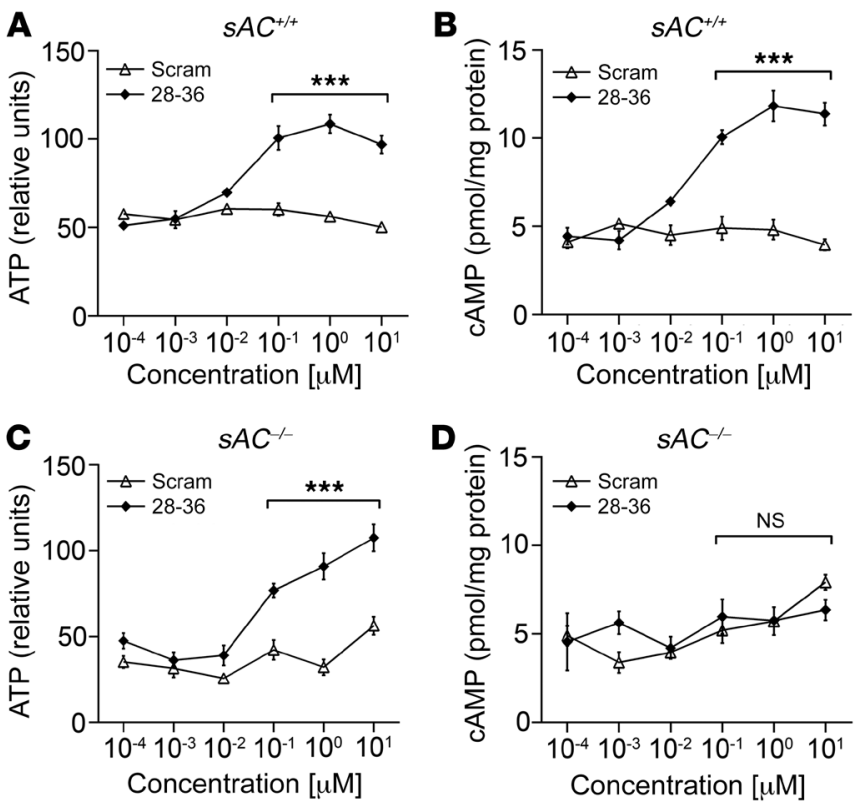

and B). Next, we knocked down cavolin-1 or clathrin in human caECs using a siRNA and observed no effect on cellular internalization of FAM(28-36) (Supplemental Figure 6, C and D). By contrast, amiloride $(1 \mathrm{mM})$, a pharmacological inhibitor of macropinocytosis (34), clearly inhibited cellular internalization of FAM(28-36) (Supplemental Figure 6, $\mathrm{E}$ and F). These experiments implicate macropinocytosis as the mechanism by which GLP-1(28-36) enters vascular cells.

We next assayed cAMP-dependent protein kinase (PKA) (27, 35), ERK (36), and eNOS (37) activations as putative downstream targets of GLP-1(28-36)-induced cAMP and/or molecules known to be involved in GLP-1-mediated cytoprotection (21). Among these, our assay for PKA revealed phosphorylation of an exogenous substrate to be 1.8- \pm 0.3 -fold higher in caSMCs exposed to GLP-1(28-36) compared with those exposed to the scrambled control $(P<0.01)$, and this phosphorylation was quantitatively similar to the effect seen with IPE (Figure 3D). Of interest, we also observed a 6-fold increase in eNOS phosphorylation in Western blotting of isolated hearts perfused with GLP-1(28-36) as compared with scramble-treated controls (Supplemental Figure 7).

To establish the functional significance of these findings, we next tested the ability of GLP-1(28-36) to prevent cytotoxicity in an in vitro model of oxidative stress (38). In this experiment, GLP-1(28-36) prevented caSMCs from cell damage (i.e., membrane integrity), as evidenced by a decreased release of LDH

Figure 5. GLP-1(28-36) does not bind SAC, but binds MTPa in mouse whole-heart lysates. (A) Western blot analysis of SAC in bound and unbound fractions from pull-down experiments using streptavidin magnetic beads with biotinylated GLP-1(28-36) and scrambled control incubated with mouse whole-heart lysates indicated no SAC binding to either peptide. SAC was, however, detected in unbound fractions. SAC - Tr, truncated active isoform of SAC protein $(50 \mathrm{kDa}) ; \mathrm{SAC}-\mathrm{FI}$, full-length isoform of SAC protein (140 kDa); WCE, whole-cell extract. (B) Western blot analysis of MTP $\alpha(\mathrm{MW}, 83 \mathrm{kDa})$ in bound and unbound fractions from identical pull-down experiments showed preferential binding of MTP $\alpha$ to biotinylated GLP-1(28-36) [BIOT(28-36)] as compared with the biotinylated scrambled(28-36) control [BIOT(scram)].
Figure 4. GLP-1(28-36) stimulates SAC-dependent intracellular CAMP accumulation in mouse caSMCs by increasing levels of the SAC substrate ATP. (A) ATP generation and (B) intracellular cAMP accumulation were analyzed as detailed in the Supplemental Methods from WT $S A C^{+/+} \mathrm{CaSMCs}$ incubated for 10 minutes with $0.1 \mathrm{nM}$ to $1 \mu \mathrm{M} \mathrm{GLP}-1(28-36)$ or scrambled control. (C) ATP generation and (D) intracellular CAMP accumulation were analyzed in $S A C^{-/}$caSMCs incubated for 10 minutes with $0.1 \mathrm{nM}$ to $1 \mu \mathrm{M}$ GLP-1(28-36) or scrambled control. GLP-1(28-36) caused a significant increase in intracellular levels of ATP at a minimum effective concentration of $0.1 \mu \mathrm{M}$ in caSMCs isolated from both WT $\left(\mathrm{SAC}^{+/+}, \mathbf{A}\right)$ and $s A C^{-1-}$ (C) mice. This effect was accompanied by a parallel accumulation of intracellular cAMP in (B) $s A C^{+/+}$caSMCs, but was lost in (D) $s A C^{-/-}$caSMCs. Data represent the mean \pm SEM. $n=3 /$ concentration/treatment group (each in triplicate). ${ }^{* * *} P<0.001$, by 2 -way ANOVA with Bonferroni's post hoc test.

(Figure 3C). Finally, the ability of GLP-1(28-36) to decrease cleaved caspase- 3 activation suggests that the agent prevents apoptosis (Figure 3E). Taken together, the above data demonstrate that GLP-1(28-36) acts through a molecular pathway involving the catalytic generation of cAMP from SAC in cardiac vascular cells to preserve membrane integrity and prevent apoptosis following oxidative injury.

GLP-1(28-36) increases cAMP levels and prevents cytotoxicity via $s A C$. To verify the mechanism of cAMP-dependent, GLP-1(28-36)mediated cytoprotection and examine the potential for translation to a clinical context, we next assessed the actions of the peptide in human caSMCs and caECs. First, human caSMCs also showed a 2-fold increase in cAMP release in response to GLP-1(28-36), which was abolished in the presence of the SAC inhibitor KH7, but not the tmAC inhibitor Ddox (Figure 2H). Mirroring data from $s A C^{-1}$ mice, depletion of sAC in human caSMCs through a siRNA also abrogated
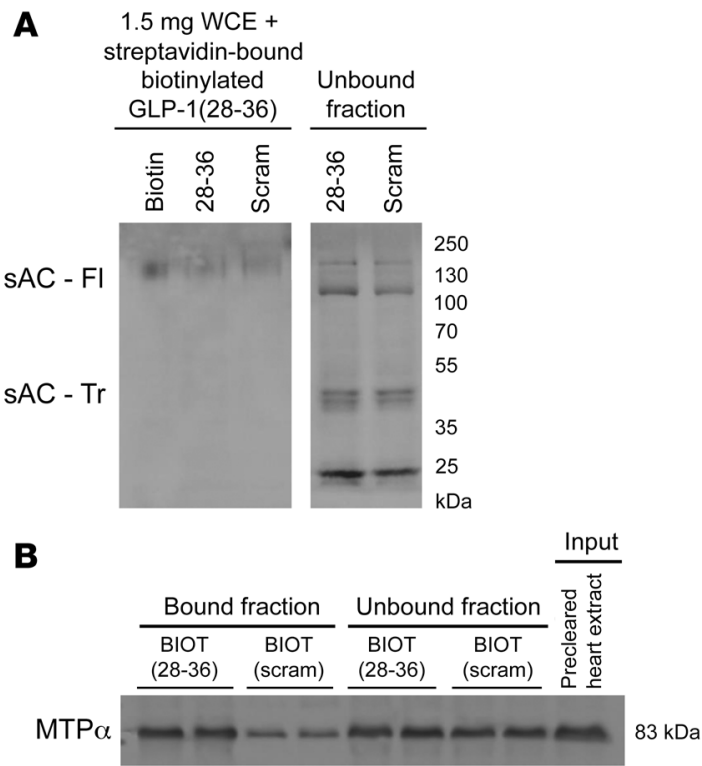

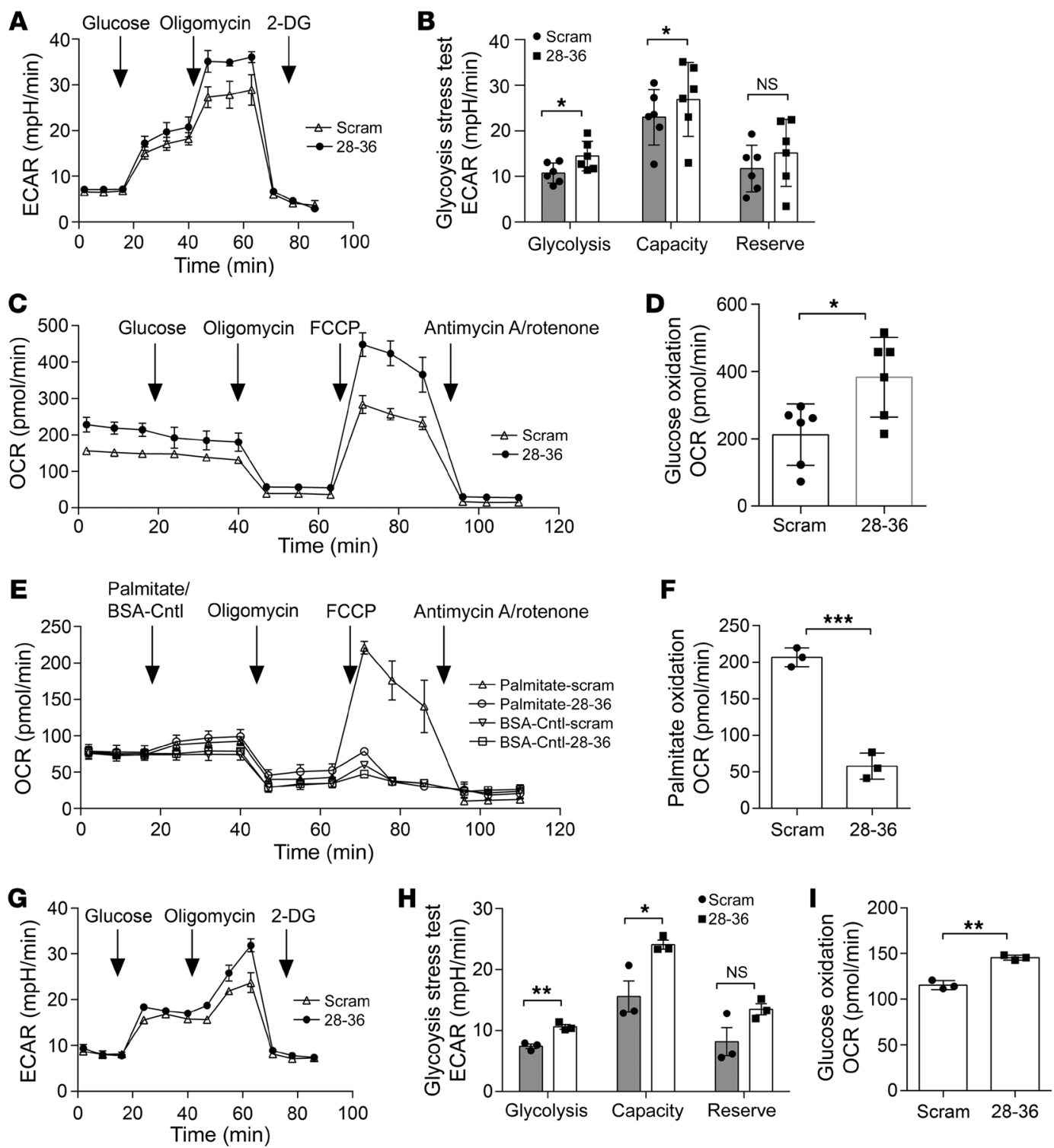

Figure 6. Pretreatment with GLP-1(28-36) increases glycolysis and glucose oxidation, and decreases FAO in coronary vascular cells. (A) Seahorse XFe24 extracellular flux analyzer traces of a glycolysis stress test in caSMCs measured as the ECAR by sequential injection of $20 \mathrm{mM}$ glucose, $1 \mu \mathrm{M}$ oligomycin, and $100 \mathrm{mM}$ 2-deoxy-D-glucose (2-DC). (B) Quantification of glycolysis, glycolytic capacity, and glycolytic reserves in caSMCs pretreated with $100 \mathrm{nM}$ GLP-1(28-36) or scrambled control for 20 minutes. (C) Seahorse XFe24 traces of glucose oxidation, measured as the OCR by sequential injection of 20 mM glucose, $1 \mu \mathrm{M}$ oligomycin, $1 \mu \mathrm{M}$ FCCP [carbonyl cyanide-4-(trifluoromethoxy)phenylhydrazone], $1 \mu \mathrm{M}$ rotenone, and $2 \mu \mathrm{M}$ antimycin A into caSMCs cultured in normal ( $5.5 \mathrm{mmol} / \mathrm{L}$ ) glucose media. (D) Quantification of glucose oxidation measured in caSMCs that were pretreated with 100 nM GLP-1(28-36) or scrambled control for 20 minutes. (E) Seahorse XFe24 traces of exogenous palmitic acid oxidation measured as the OCR following sequential injection into caSMCs of $200 \mu \mathrm{M}$ palmitic acid conjugated to BSA or BSA control, $1 \mu \mathrm{M}$ oligomycin, $1 \mu \mathrm{M}$ FCCP, $1 \mu \mathrm{M}$ rotenone, and $2 \mu \mathrm{M}$ antimycin A. (F) Quantification of

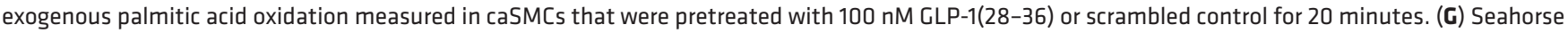
XFe24 traces from a glycolysis stress test in caECs, measured as described above. (H) Quantification of glycolysis, glycolytic capacity, and glycolytic reserves in caECs pretreated with $100 \mathrm{nM} \mathrm{GLP-1(28-36)}$ or scrambled control for 20 minutes. (I) Quantification of glucose oxidation in caECs pretreated with $100 \mathrm{nM} \mathrm{GLP-1}(28-36)$ or scrambled control for 20 minutes. $n=3$ /treatment in all Seahorse assays, each in triplicate. Data represent the mean \pm SEM. ${ }^{*} P<0.01,{ }^{* *} P<0.001$, and ${ }^{* *} P<0.0001$, by 1-way ANOVA with Bonferroni's post hoc test.

cAMP responses to GLP-1(28-36) (Figure 2I), providing further evidence of a nonreceptor, sAC-dependent mechanism. In contrast, and consistent with data from mouse ventricular CMs, we observed that GLP-1(28-36) failed to protect hESC-CMs from cytotoxicity under conditions of oxidative stress (Supplemental Figure 4C). Again, similar to the results observed in mouse caSMCs, GLP-1(28-36) activated PKA and conferred cytoprotection against oxidative stress injury in human caSMCs (Supplemental Figure 8, A and B). To investigate the importance of SAC in GLP-1(28-36)-stimulated cAMP generation in human caECs, we also blocked the anion $\left(\mathrm{HCO}_{3}\right)$ entry required for sAC activity using probenecid (39). The ability of probenecid to inhibit cAMP accumulation in GLP-1(28-36)-treated human caECs further supports our finding that GLP-1(28-36) works through SAC to increase intracellular cAMP levels (Supplemental Figure 8C). 
A

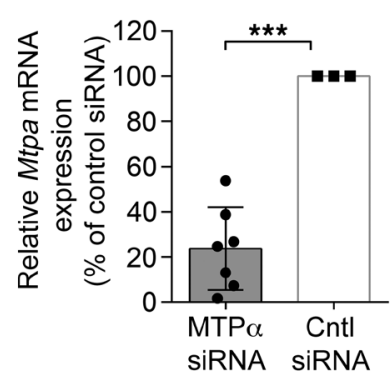

D

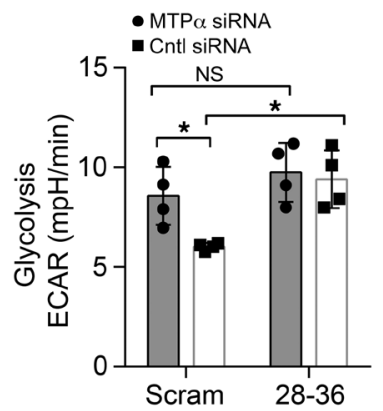

B
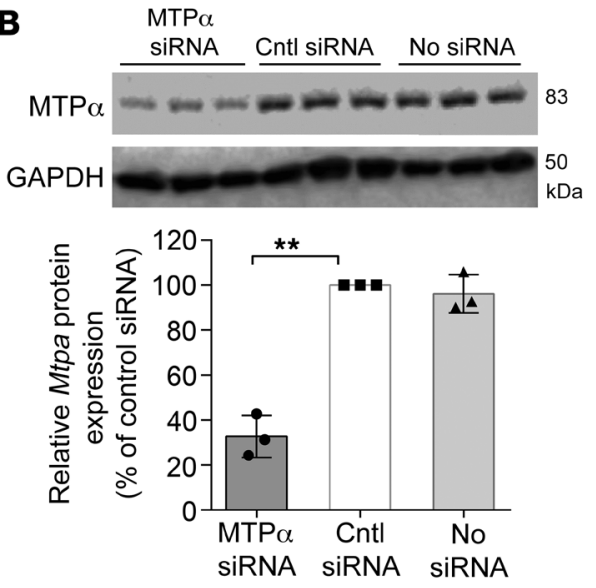

C $\quad$ mTP $\alpha$ siRna

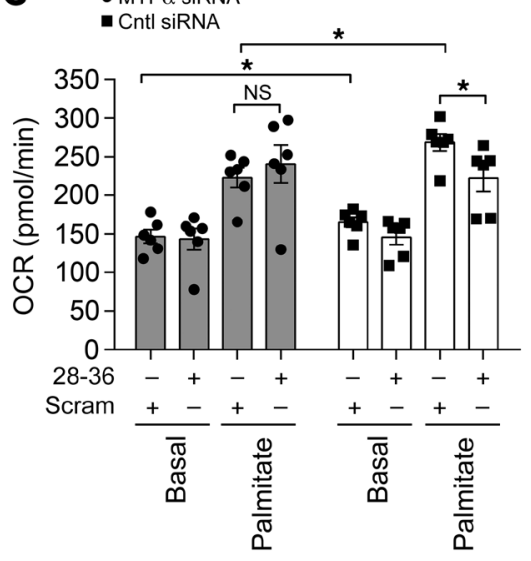

E

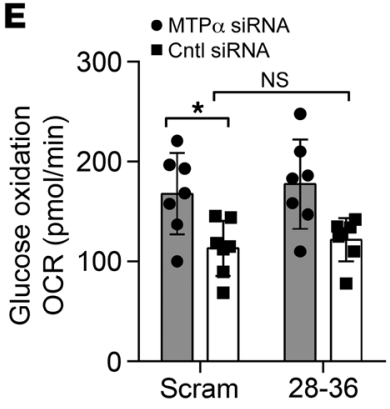

$\mathbf{F}$

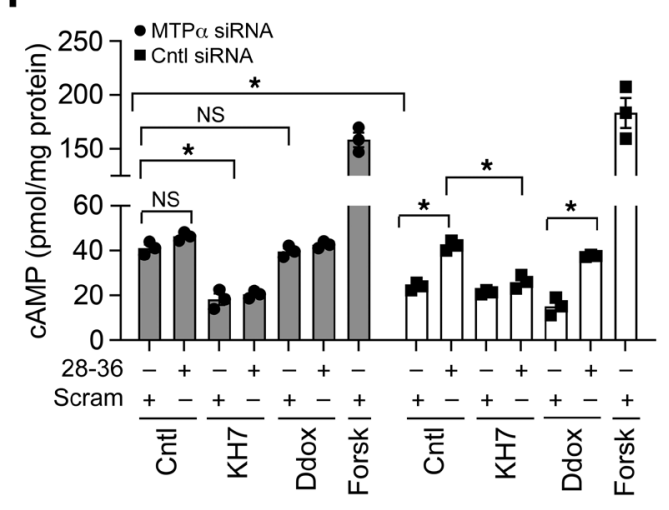

Figure 7. GLP-1(28-36) augments glucose utilization, decreases FAO, and activates sAC through MTPa. (A) Mouse caSMCs were transfected with a combination of 4 MTP $\alpha$-specific siRNAs, and relative Mtpa mRNA expression was measured by reverse transcription PCR versus control siRNA. Data were normalized to $\beta$-microglobulin and EGFP housekeeping genes (see Supplemental Methods) and are presented as a percentage of mRNA expression of the control siRNA. $n=7$ separate experiments. (B) Representative Western blot and quantitative densitometric analysis of 83-kDa MTP $\alpha$ protein expression in lysates from caSMCs transfected with MTP $\alpha$ siRNA, control siRNA, or no siRNA. Data are presented as a percentage of MTP $\alpha$ protein expression versus the loading control GAPDH $(n=3)$. (C) FAO in caSMCs was measured with a Seahorse extracellular flux analyzer as the OCR within 24 hours of transfection

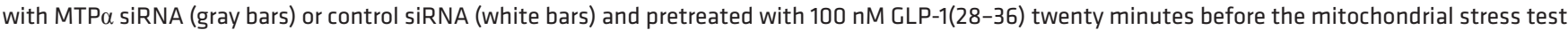
Basal respiration and palmitic acid oxidation were measured in cells transfected with MTP $\alpha$ siRNA (gray bars) or control siRNA (white bars) ( $n=7 /$ treatment, each in triplicate). (D) Glycolysis was measured as the ECAR in caSMCs treated with MTP $\alpha$ siRNA (gray bars) or control siRNA (white bars) and pre-

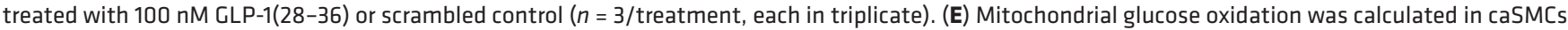

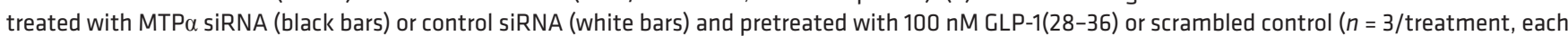
in triplicate). (F) Intracellular cAMP accumulation in MTP $\alpha$-transfected caSMCs (gray bars) and control siRNA-transfected caSMCs (white bars), shown after pretreatment with GLP-1(28-36), with or without KH7, Ddox, or forskolin. Data represent the mean $\pm \mathrm{SEM} .{ }^{*} P<0.05,{ }^{* *} P<0.01$, and ${ }^{* * *} P<0.001$, by 1-way ANOVA (A, B, D, and E) or 2-way ANOVA (C and F) with Bonferroni's post hoc test.

GLP-1(28-36) increases the levels of the sAC substrate ATP. Previous studies have reported that SAC acts as a physiological ATP sensor in glucose-responsive cells, with sAC-generated cAMP reflecting intracellular ATP levels (40). Using WT mouse caSMCs, brief, 10-minute incubations with GLP-1(28-36) caused concentration-dependent increases in ATP (Figure 4A). This effect was paralleled with increases in SAC activity, as measured by cAMP accumulation in the same lysates (Figure 4B). Importantly, although GLP-1(28-36)-driven ATP generation was retained in caSMCs isolated from $\mathrm{SAC}^{-/-}$mice (Figure 4C), cells lacking sAC could not generate cAMP in response to this stimulus (Figure 4D). These data support a mechanism whereby GLP-1(28-36) modulates SAC activity to generate cAMP in caSMCs by an increase in the ATP substrate.

GLP-1(28-36) binds MTP $\alpha$. To identify a putative mechanism by which GLP-1(28-36) stimulates ATP production, we conducted an affinity pull-down experiment with our functional biotinylated GLP-1(28-36) followed by unbiased mass spectroscopy to identify potential binding partners for GLP-1(28-36) in the mouse heart (Supplemental Figures 9-11). This analysis revealed that GLP1(28-36) did not physically interact with sAC (Figure 5A). Instead, we found that GLP-1(28-36) bound a group of inner mitochondrial membrane-localized proteins that regulate mitochondrial transport, protein binding, glycolysis, the citric acid cycle, and oxidative phosphorylation (Supplemental Table 1). More specifically, we identified MTP $\alpha$, an enzyme involved in fatty acid metabolism, as the main interacting protein of GLP-1(28-36) (Supplemental Table 2) and validated this by Western blotting (Figure $5 \mathrm{~B}$ ).

GLP-1(28-36) increases glycolysis and glucose oxidation and decreases FAO. To evaluate the effects of GLP-1(28-36) on cellular bioenergetics, we used the Seahorse extracellular flux analyzer 
A

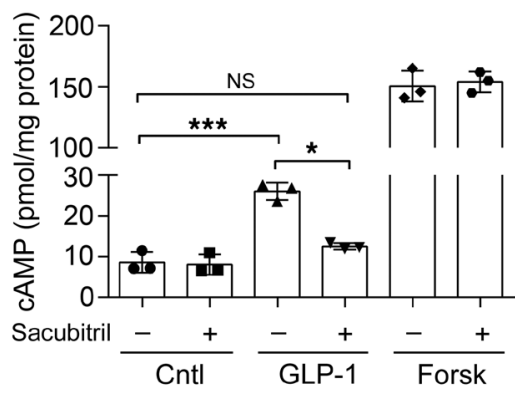

B

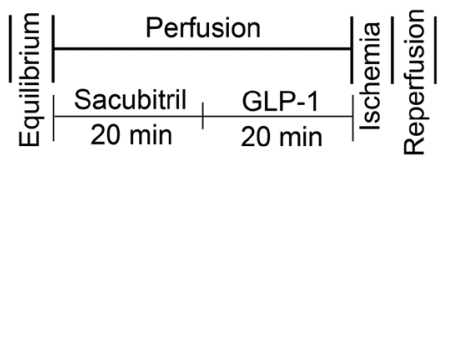

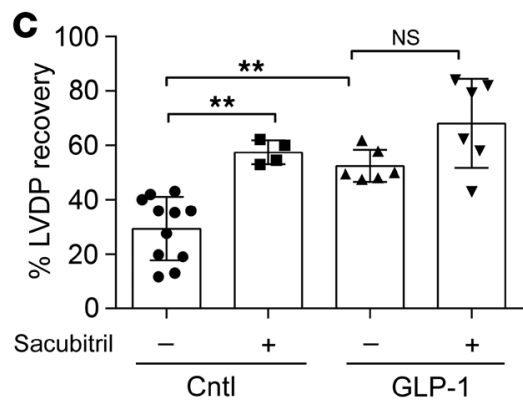

Figure 8. GLP-1(28-36) contributes to the effects of GLP-1. (A) Intracellular accumulation of cAMP was measured by enzymatic immunoassay in lysates from caSMCs treated for 10 minutes with $100 \mathrm{nM}$ each of GLP-1, forskolin, or PBS control, with or without $20 \mu \mathrm{M}$ sacubitril, a neutral endopeptidase inhibitor ( $n=3$ /treatment, each in triplicate). (B) Schematic of drug delivery and IRI protocol of Langendorff perfusion using isolated hearts from 10- to 12-week-old C57BL6] WT mice (C) LVDP recovery expressed as a percentage of LVDP at the end of reperfusion over LVDP before ischemia. LVDP recovery is shown in hearts perfused with $6 \mathrm{nM} \mathrm{GLP-1}$ or buffer-only control, with or without $1 \mu \mathrm{M}$ sacubitril ( $n=4-11 /$ group). Data represent the mean \pm SEM. ${ }^{*} P<0.05,{ }^{*} P<0.01$, and ${ }^{* * *} P<0.001$ versus the corresponding control, by 1-way ANOVA with Bonferroni's post hoc test.

and performed glycolysis stress tests to measure glycolysis and glycolytic capacity and reserve in intact caSMCs. We observed that brief pretreatment with GLP-1(28-36) versus the scrambled control increased glycolysis by $36 \%$ (extracellular acidification rate [ECAR]: $14.5 \pm 1.6$ vs. $10.7 \pm 0.7 \mathrm{mpH} / \mathrm{min} ; \mathrm{P}<0.05)$. GLP-1(28-36) also increased glycolytic capacity in caSMCs by $35 \%$ (ECAR: $30.1 \pm$ 1.5 vs. $22.3 \pm 2.6 \mathrm{mpH} / \mathrm{min} ; P<0.05$ ) (Figure 6 , A and B). We next assessed mitochondrial metabolism of glucose and palmitic acid by measuring the oxygen consumption rate (OCR). In these experiments, GLP-1(28-36) treatment increased glucose oxidation by $80 \%$ (383.5 \pm 48.3 vs. $212.2 \pm 37.2 \mathrm{pmol} / \mathrm{min} ; P<0.05$ ) (Figure 6, $\mathrm{C}$ and D) and reduced palmitic acid oxidation by $72 \%$ as compared with scramble-treated caSMCs, $(206.7 \pm 12.9$ vs. $57.9 \pm 10.5 \mathrm{pmol} /$ min; $P<0.001$ ) (Figure 6, E and F). However, GLP-1(28-36) did not affect mitochondrial glucose oxidation in caSMCs cultured in high-glucose medium (Supplemental Figure 12A).

We also assayed the bioenergetic effects of GLP-1(28-36) in caECs. GLP-1(28-36) increased glycolysis in caECs by $43 \%$ (10.6 \pm 0.4 vs. $7.4 \pm 07 \mathrm{mpH} / \mathrm{min} ; P<0.05)$ and increased their glycolytic capacity by $54 \%(24.1 \mathrm{vs.} 15.6 \pm 2.6 \mathrm{mpH} / \mathrm{min} ; P<0.05)$ (Figure $6, \mathrm{G}$ and $\mathrm{H})$. Similar to caSMCs, we found that GLP-1(28-36) increased (mitochondrial) glucose oxidation in caECs $(145.4 \pm 2.7$ vs. $115.5 \pm 2.8 \mathrm{pmol} / \mathrm{min} ; P<0.05$ ) (Figure $6 \mathrm{I}$ and Supplemental Figure 12B) without further diminishing the already low palmitic acid oxidation observed in caECs (Supplemental Figure 12C).

Energy substrate utilization and cAMP effects of GLP-1(28-36) require $M T P \alpha$. To test whether the effects of GLP-1(28-36) require its ability to interact with $\mathrm{MTP} \alpha$, we knocked down MTP $\alpha$ expression using a siRNA and repeated our Seahorse experiments. First, we documented that our siRNA approach reduced Mtpa mRNA levels by $76 \%$ and MTP $\alpha$ protein by $67 \%$ in caSMCs and compared the results with those obtained with the negative control siRNA (Figure 7, A and B). Consistent with this, MTP $\alpha$ siRNA reduced basal respiration by $15 \%$ (OCR: $143.6 \pm 6.9$ vs. $165.5 \pm 4.5 \mathrm{pmol} / \mathrm{min} ; P<0.05)$ and reduced palmitic acid-stimulated respiration by $20 \%$ (OCR: 223.3 \pm 4.5 vs. $268.8 \pm 3.3 \mathrm{pmol} / \mathrm{min} ; P<0.05)$ as compared with control siRNA-treated caSMCs. Importantly, the ability of GLP-1(28-36) to reduce palmitic acid oxidation was lost in MTP $\alpha$ siRNA-treated cells but preserved in control cells (Figure 7C).
Intriguingly, we observed a $30 \%$ increase in glycolysis and a $33 \%$ increase in mitochondrial glucose oxidation in MTP $\alpha$ siRNAversus control siRNA-treated cells (ECAR: $6.2 \pm 0.1$ vs. $8.6 \pm 0.7$ $\mathrm{mpH} / \mathrm{min}$; $P$ < 0.05; OCR: $113.4 \pm 10.1$ vs. $167.8 \pm 15.5 \mathrm{pmol} / \mathrm{min}$; $P<0.05)$. Although treatment with GLP-1(28-36) increased glycolysis by $36 \%$ in cells transfected with the control siRNA (ECAR: $6.2 \pm 0.1$ vs. $9.4 \pm 0.7 \mathrm{mpH} / \mathrm{min} ; P<0.05)$, this effect was lost in cells transfected with MTP $\alpha$ siRNA (ECAR: $8.6 \pm 0.7$ vs. $9.75 \pm$ $0.6 \mathrm{mpH} / \mathrm{min} ; P=\mathrm{NS}$ ) (Figure $7 \mathrm{D}$ ). These results indicate that GLP-1(28-36) shifts the substrate preference from fatty acid to glycolysis by inhibiting MTP $\alpha$. Again, GLP-1(28-36) did not affect mitochondrial glucose oxidation under the high-glucose concentrations used in the siRNA transfections (Figure 7E).

To explore the relationship between the MTP $\alpha$-dependent metabolic effects of GLP-1(28-36) with SAC activation and cAMP production, we knocked down MTP $\alpha$ expression in caSMCs using siRNA transfection and performed cAMP assays. MTP $\alpha$ siRNAtransfected cells showed greater intracellular cAMP accumulation than did control siRNA-transfected cells $(41.1 \pm 1.4$ vs. 24 $\pm 0.8 \mathrm{pmol} / \mathrm{mg}$ protein; $P<0.05$ ), and treatment with $100 \mathrm{nM}$ GLP-1(28-36) did not further increase cAMP levels in MTP $\alpha$ siRNA-transfected cells $(41.1 \pm 1.4$ vs. $46.3 \pm 0.8 \mathrm{pmol} / \mathrm{mg}$ protein; $P=$ NS). We further observed that the cAMP response in MTP $\alpha$ siRNA-treated cells [with or without exposure to GLP-1(28-36)] was abrogated by the sAC inhibitor KH7 [GLP-1(28-36): $41.1 \pm 1.4$ vs. $20.5 \pm 0.8 \mathrm{pmol} / \mathrm{mg}$ protein; $P<0.05$; scrambled: $41.1 \pm 1.4$ vs. $18.3 \pm 2 \mathrm{pmol} / \mathrm{mg}$ protein; $P<0.05]$, but not by the tmAC inhibitor Ddox [GLP-1(28-36): $41.1 \pm 1.4$ vs. $42.6 \pm 0.7 \mathrm{pmol} / \mathrm{mg}$ protein; $P$ = NS; scrambled: $41.1 \pm 1.4$ vs. $39.7 \pm 1.2 \mathrm{pmol} / \mathrm{mg}$ protein; $P=$ NS] (Figure 7F). As expected, a GLP-1(28-36)-induced cAMP response was preserved in control siRNA-transfected cells (42.2 \pm 1 vs. $24 \pm 0.8 \mathrm{pmol} / \mathrm{mg}$ protein; $P<0.05)$ but was lost in the control siRNA cells treated with the sAC inhibitor KH7 $(26.1 \pm 1.4 \mathrm{pmol} /$ mg protein; $P<0.05)$. Again, the tmAC inhibitor Ddox did not prevent a GLP-1(28-36)-induced cAMP response in control siRNAtreated cells $(37.6 \pm 0.2$ vs. $15.2 \pm 1.9 \mathrm{pmol} / \mathrm{mg}$ protein; $P<0.05)$ (Figure 7F). Collectively, these data reveal that GLP-1(28-36) requires $\mathrm{MTP} \alpha$-inhibitory metabolic modulation to activate sACdependent cAMP production in caSMCs. 
GLP-1(28-36) contributes to the cardiovascular biology of GLP-1. To establish the importance of the NEP-derived metabolite GLP1(28-36) to the overall cardiovascular biology of GLP-1, we used sacubitril, a pharmacological inhibitor of NEP, and assessed in vitro cAMP accumulation in caSMCs, as well as functional LVDP recovery in an ex vivo Langendorff model of IRI. We found that pretreatment with GLP-1 (100 nM) increased intracellular cAMP accumulation in caSMCs compared with untreated controls (25.9 \pm 1.6 vs. $7.6 \pm 1.1 \mathrm{pmol} / \mathrm{mg}$ protein $; P<0.0001)$ and that this effect was abolished by sacubitril $(20 \mu \mathrm{M})$, with the resulting cAMP response being no different than that of the untreated controls $(12.4 \pm 0.4$ vs. $8.4 \pm 1.3 \mathrm{pmol} / \mathrm{mg}$ protein; $P=\mathrm{NS}$ ) (Figure $8 \mathrm{~A}$ ). Next, in our isolated heart model of IRI (Figure $8 \mathrm{~B}$ ), we show that a 20-minute perfusion with GLP-1 (6 nM) improved functional LVDP recovery following IRI as compared with buffer-treated controls (52.4\% $\pm 2.7 \%$ vs. $27.6 \% \pm 4.1 \% ; P<0.05)$ (Figure $8 \mathrm{C}$ ). In contrast to our in vitro findings, the addition of sacubitril $(1 \mu \mathrm{M})$ did not abolish the improved LVDP recovery induced by GLP-1 $(52.4 \% \pm 2.7 \%$ vs. $68 \% \pm 8.1 \% ; P=\mathrm{NS}$ ) (Figure $8 \mathrm{C}$ ). However, our control isolated hearts treated with sacubitril $(1 \mu \mathrm{M})$ alone (without GLP-1) also showed improved LVDP recovery following IRI compared with the buffer-treated controls $(57.2 \% \pm 2.8 \%$ vs. $27.6 \% \pm 4.1 \% ; P<0.001$ ) (Figure $8 \mathrm{C}$ ).

\section{Discussion}

This study demonstrates that GLP-1(28-36) protects against cardiac ischemic injury. This effect of GLP-1(28-36) was independent of the known GLP-1R and was mediated by a mechanism involving mitochondrial MTP $\alpha$ and intracellular sAC in caSMCs and caECs but not CMs. We show that GLP-1(28-36) entered mouse caSMCs and ECs by macropinocytosis and activated sAC, as evidenced by the increased production of cAMP in caSMCs from WT but not $s A C^{-/-}$mice. Furthermore, GLP-1(28-36) protected mouse caSMCs from oxidative stress injury through a sAC-dependent mechanism. We also reveal that GLP-1(28-36) activated sAC by increasing production of its substrate ATP, through enhanced glucose metabolism and reduced FAO by direct inhibitory interactions with MTP $\alpha$. Finally, we show with pharmacological inhibitors (KH7) and gene silencing (siRNA) that sAC-mediated actions of GLP-1(28-36) were conserved in human caSMCs. We also observed the ability of GLP-1(28-36) to increase cAMP production through bicarbonate-responsive (i.e., probenicid-inhibited) SAC in human caECs. By contrast, there were no measurable effects of GLP-1(28-36) in hESC-CMs.

Beyond our demonstration of the effects of GLP-1(28-36) on IRI and a role for SAC in its biology, there is a small emerging body of evidence supporting a role for GLP-1(28-36) in preventing obesity, insulin resistance, diabetes mellitus, and hepatic steatosis and gluconeogenesis in mice $(11,24,27,41,42)$. This C-terminal metabolite of GLP-1 has shown cytoprotective effects in isolated mouse hepatocytes and $\beta$ cells exposed to oxidative stress injury, as indicated by trafficking to the mitochondria and the limiting of stress-induced apoptosis through inhibition of mitochondrial depolarization, cytochrome c release, and caspase activation (11, 42). These activities are consistent with our demonstration that the peptide increases ATP generation and cAMP production in a sAC-dependent manner.
Others have shown in clonal INS-1 cells and primary hepatocytes that GLP-1(28-36) increases cellular cAMP and phosphorylation of PKA, cAMP response element-binding protein (CREB), cAMPdependent transcription factor-1 (ATF-1), and $\beta$-catenin (27, 41). Just as putative roles for CREB, ATF-1, and $\beta$-catenin remain to be examined in the cardiovascular actions of GLP-1(28-36), future studies will be needed to elucidate the role of SAC in the biological actions of GLP-1(28-36) reported in hepatocytes and $\beta$ cells. Indeed, reports that $s A C^{-/-}$mice display impairments in both pancreatic insulin secretion and intraperitoneal glucose tolerance $(40,43)$ support our findings implicating sAC in GLP-1(28-36) and/or overall GLP-1 biology.

Our data also reveal an antiapoptotic role for GLP-1(28-36) through activation of SAC and its downstream cAMP-PKA effectors. However, previous findings have reported dual roles for sAC in both cell death and cell growth (44). More specifically, it has been reported that following ischemia, oxidative stress, or acidosis injury in rat caECs, CMs, and aortic SMCs, sAC-dependent apoptosis is triggered by translocation of sAC from the cytosol to mitochondria with submitochondrial PKA activation, Bax phosphorylation, and cytochrome c release (45-48). In contrast, sAC induces cell proliferation through a PKA-independent, but EPAC/ Rap1/B-Raf-dependent, signaling pathway in prostate carcinoma cells (49). Although the pro- versus antiapoptotic mechanisms of sAC are still being studied, our data support an antiapoptotic role for sAC in caSMCs and caECs. Unlike the above models, in which activation of SAC was studied after injury, we demonstrate that GLP-1(28-36) acts as a preconditioning agent by activating sAC through increased production of ATP, leading to downstream cAMP/PKA activation. Importantly, the role of PKA in activating multiple downstream targets involved in prosurvival signaling (eNOS, AKT) is well established $(4,21,50-54)$.

We previously showed that both GLP-1 and GLP-1(9-36) cause the release of guanosine $3^{\prime}, 5^{\prime}$-cyclic guanosine monophosphate (cGMP) and cAMP in coronary effluents of isolated perfused hearts from WT and Glp1r/- mice (4), thus directly implicating GLP-1 and its DPP-4-generated metabolite in GLP-1R-independent production of cAMP $(4,21)$. Others showed that both GLP-1 and DPP-4i limit infarct size in animal models of MI by increasing intracellular cAMP levels and activating PKA $(51,52)$. Indeed, preventing the degradation of cAMP by phosphodiesterase inhibition increased the infarctsparing effect of DPP-4 inhibition in mice (50). Importantly, unlike $\beta$-adrenergic stimulation-induced increases in myocardial cAMP, which enhance contractility, GLP-1 showed negative inotropic effects in rodent cardiac myocytes, implicating a compartmentalized mechanism of cAMP production away from the contractile apparatus (53). These findings provide evidence that the generation of an intracellular source of cAMP is involved in GLP-1-mediated cardioprotection.

Myocardial IRI is accompanied by several metabolic abnormalities such as increased FAO and decreased glucose oxidation (55). This phenomenon is associated with uncoupled mitochondrial respiration, elevated proton leak, ROS formation, cellular apoptosis, and, more important, increased myocardial oxygen consumption (56). Although several therapeutic strategies have attempted to reverse this metabolic imbalance (57), there is still no approved treatment regimen to date. For instance, trimetazidine (TMZ), a clinically effective antianginal agent, has been shown to improve post-IRI cardiac function in various animal models. TMZ 
was shown to modulate energy metabolism by inhabiting FAO, with a subsequent increase in glucose oxidation (58). In addition, dichloroacetic acid (DCA), a pyruvate dehydrogenase activator, enhanced cardiac efficiency during IRI of isolated rat hearts by increasing glucose oxidation (59).

Of note, Aravindhan et al. showed in rats that GLP-1(7-36) increases myocardial energy efficiency by substrate shifting to glucose (60). Although, no molecular mechanism was provided, their results are consistent with the notion that some, if not all, of the metabolic effects of the parent peptide may be mediated by our discovery that the metabolite GLP-1(28-36) inhibits MTP $\alpha$ and shifts energy substrate utilization from fatty acids to glucose. Indeed, the stimulatory effect of GLP-1(28-36) on glycolysis and its inhibitory effect on FAO were consistent in all vascular cell types studied and under all experimental conditions. Only the stimulatory effect of GLP-1(28-36) on mitochondrial glucose oxidation appeared to vary between the experimental conditions of normal and high glucose concentrations (Figure 6: $5.5 \mathrm{mmol} / \mathrm{L}$; Figure 7 and Supplemental Figure 12A: $20 \mathrm{mmol} / \mathrm{L}$ ).

Affinity pull-down and mass spectrometric (MS) analyses consistently identified MTP $\alpha$ (also known as HADHA) as the main interacting binding partner for GLP-1(28-36). However, this process revealed additional binding partners including proteins that regulate oxidative phosphorylation, mitochondrial membrane transport, the citric acid cycle, and glycolysis (Supplemental Table 1). Although functional Seahorse assays clearly confirmed a role for MTP $\alpha$ in mediating the ability to modulate cellular metabolism, we cannot exclude the possibility that GLP-1(28-36) may also interact with other partner proteins to achieve its effects on cell metabolism and cytoprotection.

In this study, we did not observe significant increases in extracellular cAMP release in coronary effluent from GLP-1(28-36)perfused mouse hearts (Figure 2B) as compared with robust responses in caSMCs and caECs in vitro (Figure 3, A and B). We speculate that the molecular actions of GLP-1(28-36) are localized intracellularly to specific cardiovascular cell types. Although CMs have long been implicated as the main cellular targets of cardioprotective agents against MI and/or IRI, our in vitro data reveal that CMs are not direct sites of action of the cytoprotective effects of GLP-1(28-36). However, studies examining the specific role of cardiac and/or coronary vascular cells in IRI are scarce. To our knowledge, the strategy of selective prevention of cytotoxicity of cardiac vascular cells following MI and/or IRI to affect whole-organ cardioprotection has never been directly studied. Although our data suggest that such an approach is feasible, further work is required to determine the relative importance of cardiac vascular cells versus cardiac myocytes in the pathophysiology of IRI. For example, tissue-specific KOs of sAC (e.g., SMCs vs. ECs vs. CMs) may be required to parse the cell-specific role of this cAMP-generating pathway in IRI, with the lack of GLP-1(28-36) responses in $s A C^{-1-}$ caSMCs providing a strong rationale.

In attempting to understand whether manipulation of NEP24.11 to prevent the degradation of GLP-1 into GLP-1(28-36) would affect the clinically observed cardioprotective actions of GLP-1, we found that the in vitro cAMP response to GLP-1 was lost with addition of the NEP24.11 inhibitor sacubitril. However, sacubitril failed to inhibit the functional LVDP recovery observed with GLP-1 in our ex vivo model of IRI. We believe this may be due to pleiotropic actions of NEP24.11 and the activation of GLP-1unrelated cardioprotective signaling molecules (e.g., ANP) in the more complex ex vivo system. Because of this, carefully designed experiments with NEP21.11 and ANP and/or NRP-1 knockdowns may be required to further parse the role of GLP-1(28-36) in the overall cardiovascular biology of GLP-1. Indeed, it is not yet known whether GLP-1(28-36) is a physiologically relevant, endogenously active metabolite. We are in the process of developing MS methods for quantitative analysis of GLP-1(28-36) and have developed a MS signature using synthetic GLP-1(28-36) peptide at the MS retention time of 17.74 minutes; data not shown). Assays for the in vivo measurement of plasma levels and tissue activity of GLP-1(28-36) and subsequent functional analysis will be pivotal to understanding the physiological relevance of this peptide.

A large cardiovascular outcome trial in high-risk T2D patients treated with the GLP-1 analog liraglutide (LEADER) demonstrated impressive reductions in CV mortality $(-22 \% ; P<0.001)$, with nonsignificant reductions in hospitalizations for heart failure (hHF) $(-13 \% ; P=0.14)(13)$. Interestingly, these benefits may be blunted in the approximately $30 \%$ of patients in whom liraglutide increased the resting heart rate (HR) by $10 \mathrm{bpm}$ or more (61). Furthermore, in smaller studies of patients with recent acute and advanced HF, irrespective of T2D status $(62,63)$, treatment with liraglutide was associated with a nonsignificant increase in CV mortality and hHF $(30 \%$, $P=0.17$ ) (62). It is tempting to speculate that this apparent loss of benefit in patients with more advanced HF may be the result of increases in HR caused by activation of sinoatrial node (SAN) GLP-1Rs (64, 65), which could be mitigated with an agent such as GLP-1(28-36) that retains cardiac and vascular protection of GLP-1, without activating the GLP-1R. Indeed, the potential promise of GLP-1(28-36) as a synthetic peptide that enables GLP-1R-independent, MTP $\alpha$ dependent, sAC-mediated cytoprotective cAMP signaling, with no effect on HR, is worthy of further exploration.

In conclusion, we have identified molecular and cellular targets for GLP-1(28-36) in the cardiovascular system. We believe that the mechanistic pathways uncovered here have potential for impact in the clinical context of IRI, for which no approved therapies currently exist.

\section{Methods}

Animals. The origins and specifications of the mice used in this study are detailed in the Supplemental Methods.

Descriptions and sources of reagents and detailed methods of our mouse models of experimental MI in vivo (66), IRI in vivo (67), IRI ex vivo $(4,21)$, as well as mouse and human cellular injury models in vitro $(21,68)$ are provided in the Supplemental Methods. Details on the isolation of mouse caSMCs and neonatal ventricular CMs, as well as cell culture methods for human caSMCs, caECs, hESC-CMs, and the HL-1 cell line, including drug treatment regimens, are provided in the Supplemental Methods. All assays relevant to cAMP, LDH, PKA, ATP and Seahorse XFe24 extracellular flux measurements are detailed. Gene silencing using siRNA and Western blot protocols are described in the Supplemental Methods, as is the use of streptavidin affinity pull-down of biotinylated peptides and MS.

Statistics. Data are expressed as the mean \pm SEM. A 1-way ANOVA was used to analyze differences between 3 or more treatment groups using GraphPad Prism, version 4.0 (GraphPad Software). A 2-way 
ANOVA was used to compare differences between GLP-1(28-36) and the scrambled control across multiple doses or different mouse strains. Bonferroni's post hoc test was used for multiple comparisons. A $P$ value of less than 0.05 was considered significant.

\section{Author contributions}

DM, MAS, TA, YL, WL, and MH conceived and designed the experiments. DM, MAS, SB, AM, TA, XS, YL, and SG conducted the experiments and analyzed the data. GK, MBW, and PB provided access to key reagents and experimental core facilities. DM, MAS, EAS, TA, and $\mathrm{MH}$ wrote and revised the manuscript. $\mathrm{MH}$ is the guarantor of this work and, as such, had full access to data from the study and takes responsibility for the integrity of the data and accuracy of the data analysis.

\section{Acknowledgments}

We thank Daniel Drucker and Anthony Gramolini (Toronto, Canada) and Gary Lopaschuk (Edmonton, Canada) for helpful discussions and Daniel Drucker for providing the Glp1r/- mice. This study was funded by operating grants and salary support to $\mathrm{MH}$ from the Heart and Stroke Foundation of Ontario (HSFO) (grants T6757, NA6997, and CI6824) and the Canadian Institutes of Health Research (CIHR) (grant PJT 162205).

Address correspondence to: Mansoor Husain, Toronto General Hospital Research Institute, 101 College Street, TMDT Room 3-909, Toronto, Ontario, M5G 1L7, Canada. Phone: 416.634.8254; Email: mansoor.husain@utoronto.ca.
1. Herlitz J, Karlson BW, Hjalmarson A. Ten year mortality in relation to original size of myocardial infarct: results from the Gothenburg metoprolol study. Br Heart J. 1994;71(3):238-241.

2. Yellon DM, Hausenloy DJ. Myocardial reperfusion injury. N Engl J Med. 2007;357(11):1121-1135.

3. Hausenloy DJ, Yellon DM. Myocardial ischemia-reperfusion injury: a neglected therapeutic target. J Clin Invest. 2013;123(1):92-100.

4. Ban K, Noyan-Ashraf MH, Hoefer J, Bolz SS, Drucker DJ, Husain M. Cardioprotective and vasodilatory actions of glucagon-like peptide 1 receptor are mediated through both glucagon-like peptide 1 receptor-dependent and -independent pathways. Circulation. 2008;117(18):2340-2350.

5. Nikolaidis LA, et al. Recombinant glucagon-like peptide-1 increases myocardial glucose uptake and improves left ventricular performance in conscious dogs with pacing-induced dilated cardiomyopathy. Circulation. 2004;110(8):955-961.

6. Sonne DP, Engstrøm T, Treiman M. Protective effects of GLP-1 analogues exendin-4 and GLP1(9-36) amide against ischemia-reperfusion injury in rat heart. Regul Pept. 2008;146(1-3):243-249.

7. Timmers L, et al. Exenatide reduces infarct size and improves cardiac function in a porcine model of ischemia and reperfusion injury. J Am Coll Cardiol. 2009;53(6):501-510.

8. Lønborg J, et al. Exenatide reduces final infarct size in patients with ST-segment-elevation myocardial infarction and short-duration of ischemia. Circ Cardiovasc Interv. 2012;5(2):288-295.

9. Read PA, et al. A pilot study to assess whether glucagon-like peptide-1 protects the heart from ischemic dysfunction and attenuates stunning after coronary balloon occlusion in humans. Circ Cardiovasc Interv. 2011;4(3):266-272.

10. Mentlein R, Gallwitz B, Schmidt WE. Dipeptidyl-peptidase IV hydrolyses gastric inhibitory polypeptide, glucagon-like peptide-1(7-36) amide, peptide histidine methionine and is responsible for their degradation in human serum. Eur J Biochem. 1993;214(3):829-835

11. Tomas E, Stanojevic V, Habener JF. GLP-1derived nonapeptide GLP-1(28-36)amide targets to mitochondria and suppresses glucose production and oxidative stress in isolated mouse hepatocytes. Regul Pept. 2011;167(2-3):177-184.

12. Noyan-Ashraf MH, et al. A glucagon-like pep- tide- 1 analog reverses the molecular pathology and cardiac dysfunction of a mouse model of obesity. Circulation. 2013;127(1):74-85.

13. Marso SP, et al. Liraglutide and cardiovascular outcomes in type 2 diabetes. $N$ Engl J Med. 2016;375(4):311-322.

14. Husain M, et al. Oral semaglutide and cardiovascular outcomes in patients with type 2 diabetes. N Engl J Med. 2019;381(9):841-851.

15. Gerstein HC, et al. Dulaglutide and cardiovascular outcomes in type 2 diabetes (REWIND): a double-blind, randomised placebo-controlled trial. Lancet. 2019;394(10193):121-130.

16. Hernandez AF, et al. Albiglutide and cardiovascular outcomes in patients with type 2 diabetes and cardiovascular disease (Harmony Outcomes): a double-blind, randomised placebo-controlled trial. Lancet. 2018;392(10157):1519-1529.

17. Pfeffer MA, et al. Lixisenatide in patients with type 2 diabetes and acute coronary syndrome. NEngl JMed. 2015;373(23):2247-2257.

18. Holman RR, et al. Effects of once-weekly exenatide on cardiovascular outcomes in type 2 diabetes. N Engl J Med. 2017;377(13):1228-1239.

19. Nikolaidis LA, et al. Effects of glucagon-like peptide- 1 in patients with acute myocardial infarction and left ventricular dysfunction after successful reperfusion. Circulation. 2004;109(8):962-965.

20. Ussher JR, Drucker DJ. Cardiovascular biology of the incretin system. Endocr Rev. 2012;33(2):187-215

21. Ban K, et al. Glucagon-like peptide (GLP)1(9-36)amide-mediated cytoprotection is blocked by exendin(9-39) yet does not require the known GLP-1 receptor. Endocrinology. 2010;151(4):1520-1531.

22. Ussher JR, et al. Inactivation of the cardiomyocyte glucagon-like peptide-1 receptor (GLP1R) unmasks cardiomyocyte-independent GLP-1R-mediated cardioprotection. Mol Metab. 2014;3(5):507-517.

23. Jackson S, et al. Combined enzyme defect of mitochondrial fatty acid oxidation. J Clin Invest. 1992;90(4):1219-1225.

24. Tomas E, Wood JA, Stanojevic V, Habener JF. GLP-1-derived nonapeptide GLP-1(28-36)amide inhibits weight gain and attenuates diabetes and hepatic steatosis in diet-induced obese mice. Regul Pept. 2011;169(1-3):43-48.
25. Noyan-Ashraf MH, et al. GLP-1R agonist liraglutide activates cytoprotective pathways and improves outcomes after experimental myocardial infarction in mice. Diabetes. 2009;58(4):975-983.

26. Gros R, et al. Cardiac function in mice lacking the glucagon-like peptide-1 receptor. Endocrinology. 2003;144(6):2242-2252.

27. Shao W, et al. GLP-1(28-36) improves $\beta$-cell mass and glucose disposal in streptozotocin-induced diabetic mice and activates cAMP/PKA/ $\beta$-catenin signaling in $\beta$-cells in vitro. Am J Physiol Endocrinol Metab. 2013;304(12):E1263-E1272.

28 . Sanada S, et al. Protein kinase A as another mediator of ischemic preconditioning independent of protein kinase C. Circulation. 2004;110(1):51-57.

29. Chen Y, et al. Soluble adenylyl cyclase as an evolutionarily conserved bicarbonate sensor. Science. 2000;289(5479):625-628.

30. Pierre S, Eschenhagen T, Geisslinger G, Scholich K. Capturing adenylyl cyclases as potential drug targets. Nat Rev Drug Discov. 2009;8(4):321-335.

31. Qa'aty $\mathrm{N}$, et al. The antidiabetic hormone glucagon-like peptide-1 induces formation of new elastic fibers in human cardiac fibroblasts after cross-activation of IGF-IR. Endocrinology. 2015;156(1):90-102.

32. Cameron-Vendrig A, Mundil D, Husain M. Antiatherothrombotic effects of dipeptidyl peptidase inhibitors. Curr Atheroscler Rep. 2014;16(5):408.

33. McCluskey A, et al. Building a better dynasore: the dyngo compounds potently inhibit dynamin and endocytosis. Traffic. 2013;14(12):1272-1289.

34. Koivusalo M, et al. Amiloride inhibits macropinocytosis by lowering submembranous $\mathrm{pH}$ and preventing Rac1 and Cdc42 signaling. J Cell Biol. 2010;188(4):547-563.

35. Dohi T, Xia F, Altieri DC. Compartmentalized phosphorylation of IAP by protein kinase A regulates cytoprotection. Mol Cell. 2007;27(1):17-28.

36. Huang Y, et al. An alpha1A-adrenergic-extracellular signal-regulated kinase survival signaling pathway in cardiac myocytes. Circulation. 2007;115(6):763-772.

37. Prorock AJ, Hafezi-Moghadam A, Laubach VE, Liao JK, Ley K. Vascular protection by estrogen in ischemia-reperfusion injury requires endothelial nitric oxide synthase. Am J Physiol Heart Circ Physiol. 2003;284(1):H133-H140.

38. Nishida M, Maruyama Y, Tanaka R, Kontani K, Nagao T, Kurose H. G alpha(i) and G alpha(o) 
are target proteins of reactive oxygen species. Nature. 2000;408(6811):492-495.

39. Osycka-Salut C, et al. Cyclic AMP efflux, via MRPs and $\mathrm{A} 1$ adenosine receptors, is critical for bovine sperm capacitation. Mol Hum Reprod. 2014;20(1):89-99.

40. Zippin JH, et al. $\mathrm{CO}_{2} / \mathrm{HCO}_{3}^{-}$- and calciumregulated soluble adenylyl cyclase as a physiological ATP sensor. J Biol Chem. 2013;288(46):33283-33291.

41. Ip W, Shao W, Chiang YT, Jin T. GLP-1-derived nonapeptide GLP-1(28-36)amide represses hepatic gluconeogenic gene expression and improves pyruvate tolerance in high-fat diet-fed mice. Am J Physiol Endocrinol Metab. 2013;305(11):E1348-E1358.

42. Liu Z, Stanojevic V, Brindamour LJ, Habener JF. GLP1-derived nonapeptide GLP1(28-36)amide protects pancreatic $\beta$-cells from glucolipotoxicity. J Endocrinol. 2012;213(2):143-154.

43. Holz GG, Leech CA, Chepurny OG. New insights concerning the molecular basis for defective glucoregulation in soluble adenylyl cyclase knockout mice. Biochim Biophys Acta. 2014;1842(12 Pt B):2593-2600.

44. Ladilov Y, Appukuttan A. Role of soluble adenylyl cyclase in cell death and growth. Biochim Biophys Acta. 2014;1842(12 Pt B):2646-2655.

45. Appukuttan A, Kasseckert SA, Kumar S, Reusch HP, Ladilov Y. Oxysterol-induced apoptosis of smooth muscle cells is under the control of a soluble adenylyl cyclase. Cardiovasc Res. 2013;99(4):734-742.

46. Appukuttan A, et al. Type 10 adenylyl cyclase mediates mitochondrial Bax translocation and apoptosis of adult rat cardiomyocytes under simulated ischaemia/reperfusion. Cardiovasc Res. 2012;93(2):340-349.

47. Kumar S, Appukuttan A, Maghnouj A, Hahn S, Peter Reusch H, Ladilov Y. Suppression of soluble adenylyl cyclase protects smooth muscle cells against oxidative stress-induced apoptosis. Apoptosis. 2014;19(7):1069-1079.

48. Kumar S, Kostin S, Flacke JP, Reusch HP, Ladilov Y. Soluble adenylyl cyclase controls mitochondria-dependent apoptosis in coronary endothelial cells. J Biol Chem. 2009;284(22):14760-14768.
49. Flacke JP, et al. Type 10 soluble adenylyl cyclase is overexpressed in prostate carcinoma and controls proliferation of prostate cancer cells. J Biol Chem. 2013;288(5):3126-3135.

50. Birnbaum Y, et al. Phosphodiesterase III inhibition increases cAMP levels and augments the infarct size limiting effect of a DPP-4 inhibitor in mice with type-2 diabetes mellitus. Cardiovasc Drugs Ther. 2012;26(6):445-456.

51. Bose AK, Mocanu MM, Carr RD, Brand CL, Yellon DM. Glucagon-like peptide 1 can directly protect the heart against ischemia/reperfusion injury. Diabetes. 2005;54(1):146-151.

52. Ye Y, Keyes KT, Zhang C, Perez-Polo JR, Lin Y, Birnbaum Y. The myocardial infarct size-limiting effect of sitagliptin is PKA-dependent, whereas the protective effect of pioglitazone is partially dependent on PKA. Am J Physiol Heart Circ Physiol. 2010;298(5):H1454-H1465.

53. Vila Petroff MG, Egan JM, Wang X, Sollott SJ. Glucagon-like peptide-1 increases cAMP but fails to augment contraction in adult rat cardiac myocytes. Circ Res. 2001;89(5):445-452.

54. Yang C, Talukder MA, Varadharaj S, Velayutham M, Zweier JL. Early ischaemic preconditioning requires Akt- and PKA-mediated activation of eNOS via serine1176 phosphorylation. Cardiovasc Res. 2013;97(1):33-43.

55. Fillmore N, Mori J, Lopaschuk GD. Mitochondrial fatty acid oxidation alterations in heart failure, ischaemic heart disease and diabetic cardiomyopathy. Br J Pharmacol. 2014;171(8):2080-2090.

56. Boudina S, Abel ED. Mitochondrial uncoupling: a key contributor to reduced cardiac efficiency in diabetes. Physiology (Bethesda). 2006;21:250-258.

57. Jaswal JS, Keung W, Wang W, Ussher JR, Lopaschuk GD. Targeting fatty acid and carbohydrate oxidation--a novel therapeutic intervention in the ischemic and failing heart. Biochim Biophys Acta. 2011;1813(7):1333-1350.

58. Kantor PF, Lucien A, Kozak R, Lopaschuk GD. The antianginal drug trimetazidine shifts cardiac energy metabolism from fatty acid oxidation to glucose oxidation by inhibiting mitochondrial long-chain 3-ketoacyl coenzyme A thiolase. Circ Res. 2000;86(5):580-588.

59. Taniguchi M, Wilson C, Hunter CA, Pehowich
DJ, Clanachan AS, Lopaschuk GD. Dichloroacetate improves cardiac efficiency after ischemia independent of changes in mitochondrial proton leak. Am J Physiol Heart Circ Physiol. 2001;280(4):H1762-H1769.

60. Aravindhan K, Bao W, Harpel MR, Willette RN, Lepore JJ, Jucker BM. Cardioprotection resulting from glucagon-like peptide-1 administration involves shifting metabolic substrate utilization to increase energy efficiency in the rat heart. PLoS One. 2015;10(6):e0130894.

61. Husain M, et al. Arrythmias and heart rate increase in the LEADER trial and relation to the risk of cardiovascular events. Eur Heart J. 2018;39(suppl_1):ehy565.P2518.

62. Margulies KB, et al. Effects of liraglutide on clinical stability among patients with advanced heart failure and reduced ejection fraction: a randomized clinical trial. JAMA. 2016;316(5):500-508.

63. Jorsal A, et al. Effect of liraglutide, a glucagonlike peptide-1 analogue, on left ventricular function in stable chronic heart failure patients with and without diabetes (LIVE)-a multicentre, double-blind, randomised, placebo-controlled trial. Eur J Heart Fail. 2017;19(1):69-77.

64. Lorenz M, et al. Differential effects of glucagon-like peptide-1 receptor agonists on heart rate. Cardiovasc Diabetol. 2017;16(1):6.

65. Pyke C, et al. GLP-1 receptor localization in monkey and human tissue: novel distribution revealed with extensively validated monoclonal antibody. Endocrinology. 2014;155(4):1280-1290.

66. Zaidi SH, Huang Q, Momen A, Riazi A, Husain M. Growth differentiation factor 5 regulates cardiac repair after myocardial infarction. J Am Coll Cardiol. 2010;55(2):135-143.

67. Xu Z, Alloush J, Beck E, Weisleder N. A murine model of myocardial ischemia-reperfusion injury through ligation of the left anterior descending artery. J Vis Exp. 2014;(86):e51329.

68. Sun X, et al. p27 protein protects metabolically stressed cardiomyocytes from apoptosis by promoting autophagy. J Biol Chem . 2014;289(24):16924-16935. 